ARTICLE

DOI: $10.1038 / \mathrm{s} 41467-018-05182-9$

\title{
Endogenous transcripts control miRNA levels and activity in mammalian cells by target-directed miRNA degradation
}

Francesco Ghini ${ }^{1}$, Carmela Rubolino ${ }^{1}$, Montserrat Climent ${ }^{1}$, Ines Simeone ${ }^{1}$, Matteo J. Marzi ${ }^{1} \&$ Francesco Nicassio (1) ${ }^{1}$

Little is known about miRNA decay. A target-directed miRNA degradation mechanism (TDMD) has been suggested, but further investigation on endogenous targets is necessary. Here, we identify hundreds of targets eligible for TDMD and show that an endogenous RNA (Serpine1) controls the degradation of two miRNAs (miR-30b-5p and miR-30c-5p) in mouse fibroblasts. In our study, TDMD occurs when the target is expressed at relatively low levels, similar in range to those of its miRNAs (100-200 copies per cell), and becomes more effective at high target:miRNA ratios (>10:1). We employ CRISPR/Cas9 to delete the miR-30 responsive element within Serpine1 3'UTR and interfere with TDMD. TDMD suppression increases miR-30b/c levels and boosts their activity towards other targets, modulating gene expression and cellular phenotypes (i.e., cell cycle re-entry and apoptosis). In conclusion, a sophisticated regulatory layer of miRNA and gene expression mediated by specific endogenous targets exists in mammalian cells.

\footnotetext{
${ }^{1}$ Center for Genomic Science of IIT@SEMM, Istituto Italiano di Tecnologia (IIT)_Via Adamello 16, 20139 Milan, Italy. These authors contributed equally: Francesco Ghini, Carmela Rubolino. These authors jointly supervised this work: Matteo J. Marzi, Francesco Nicassio. Correspondence and requests for materials should be addressed to M.J.M. (email: matteo.marzi@iit.it) or to F.N. (email: francesco.nicassio@iit.it)
} 
$\mathrm{M}$ icroRNAs (miRNAs) are an evolutionarily conserved class of small (about 18-22 nt long) non-coding RNAs that function in post-transcriptional regulation of gene expression ${ }^{1}$. Targets are bound through base paring between the miRNA and their miRNA responsive elements (MREs), usually located in the $3^{\prime}$ untranslated region $\left(3^{\prime} \mathrm{UTR}\right)^{2}$. To act as such, any MRE usually presents complementarity to bases 2-7 (the seed) of miRNAs; however, other sequences, usually located near the miRNA $3^{\prime}$ end, may also form additional base pairs and thus participate in target recognition. Due to the low levels of complementarity between miRNAs and their RNA targets, from hundreds to thousands RNAs could interact with the same miRNA sequence, as demonstrated by high-throughput experimental studies ${ }^{3,4}$. For the interaction with their targets to take place, miRNAs must be loaded onto Argonaute proteins (AGO) and form the core of the RNA-induced silencing complex (RISC). Within RISC, miRNAs induce silencing by target destabilisation and/or translational repression ${ }^{5,6}$. Computational methods, such as TargetScan ${ }^{7}$ and others 8 , are able to predict miRNA targets and their MREs based on seed type hierarchy (8-mer $>7$ mer-m8 $>7$-mer-A1 $>6$-mer) and on sequence conservation of orthologous mRNAs as found by comparative genome analysis. Usually, target expression changes slightly when miRNA levels are perturbed ${ }^{9,10}$; however, the resulting phenotypic effect can be profound as targets often converge towards the same pathway or biological process.

Intriguingly, target:miRNA interactions have been suggested to act as a bidirectional control mechanism, with targets in turn affecting miRNAs activity. Two mechanisms have been reported: the competing endogenous RNA (ceRNA) hypothesis ${ }^{11}$ and the target-directed miRNA degradation (TDMD) mechanism ${ }^{12}$. The ceRNA theory postulates that endogenous targets compete with each other for binding to a shared miRNA; therefore, a sudden change in the expression of a competing endogenous target (e.g., the ceRNA) might influence miRNA activity on other targets $^{13}$. Most evidence in favour of the ceRNA hypothesis comes from over-expression approaches, so that the impact of ceRNAs on miRNA-mediated mechanisms in physiological settings is still debated $^{14-16}$. In the TDMD mechanism, the RNA target (the TDMD target) promotes degradation of its miRNA ${ }^{17,18}$, accompanied by post-transcriptional modification of the miRNA sequence, i.e., tailing (addition of nucleotides at the $3^{\prime}$ end) and trimming (shortening) ${ }^{19}$, and unloading from $\mathrm{AGO}^{20}$. Studies performed using artificial targets showed that extended complementarity to miRNAs $3^{\prime}$ regions combined with a central bulge of $\leq 5 \mathrm{nt}$, promotes miRNA degradation ${ }^{18,21}$. However, TDMD molecular basis and physiological role are still obscure. Endogenous RNA targets implicated in TDMD and the role they play in modulating miRNA activity need to be further investigated, especially in non-neuronal cells. So far, the evidence for accelerated miRNA decay comes from studies on viral targets (e.g., the non-coding HSUR RNA and m169 mRNA $^{22,23}$ ) and on artificial transcripts, both characterised either by a central bulge or by perfect complementarity ${ }^{15,24}$. Indeed, it has been shown that, in physiological conditions, miRNA decay can be accelerated by a rapid change in gene expression (e.g., light-dark transition or growth factor stimulation ${ }^{25,26}$ ), suggesting the existence of a posttranscriptional mechanism able to control miRNA levels. However, precise molecular details remain obscure. We and others have recently shed light on the dynamics of miRNA decay in mammalian cells by using new tailored approaches based on in vivo RNA labelling 27,28 . In our study, different pools of miRNAs were identified on the basis of their decay pattern: "slow" miRNAs, very stable $\left(T_{1 / 2}>24 \mathrm{~h}\right)$, and apparently downregulated through dilution by cell division; and "fast" miRNAs, quickly turned over in the cell ( $T_{1 / 2}$ from 4 to $\left.14 \mathrm{~h}\right)$. Intriguingly, we found a significant association between miRNA type of decay ("fast" or "slow") and number and expression levels of targets bearing an additional complementarity to miRNA $3^{\prime}$ ends (3C$\operatorname{targets}^{27}$ ), as required for TDMD. On these bases, we hypothesised that TDMD is a general mechanism, with specific endogenous targets able to control the dynamics of degradation of their miRNAs.

In this study, we provide evidence that the endogenous target Serpinel is able to control the levels of the two miRNAs miR-30b$5 p$ and $-30 c-5 p$ by inducing their degradation during cell cycle reentry of quiescent fibroblasts stimulated with serum. Following an in-depth investigation of the interaction between Serpinel and $\mathrm{miR}-30 \mathrm{~b} / \mathrm{c}$, we describe TDMD as a post-transcriptional mechanism that controls levels and activity of miRNAs.

\section{Results}

Candidate decay targets are present in the mammalian genome. Using the TargetScan database as a source of miRNA:target pairs, we identified endogenous RNAs that could trigger miRNA degradation and focused our analysis on targets presenting a $3^{\prime}$ pairing contribution (3C-score ${ }^{29}$ ) on top of the canonical seed pairing (3C-targets, Fig. 1a and Supplementary Data 1). Overall, nearly $15 \%$ of all the target:miRNA predicted interactions involved a $3 \mathrm{C}$ site. However, most of these interactions were characterised by limited complementarity (e.g., three or four base pairs) at the miRNA $3^{\prime}$ end, which, according to previous studies with artificial targets ${ }^{18,20,21}$, should not be sufficient for triggering TDMD. Based on their 3C-scores, 3C-targets were divided into three classes: (i) low, with 3C-scores between -0.01 and -0.03 ; (ii) mid, scoring between -0.03 and -0.05 ; and (iii) high, with 3Cscore lower than -0.05 (examples in Fig. 1b). The 3C-high class, which is characterised by the highest complementarity, included only a minority of all the predicted interactions $(n=17988,0.8 \%$; Fig. 1c and Supplementary Fig. 1). In addition, the likelihood of interaction between a miRNA and a given target (and the effects of such an interaction) also depends on their relative expression levels ${ }^{14,15}$. Hence, the repertoire of $3 \mathrm{C}$ interactions that could affect miRNA stability is strictly context-dependent. We focused on mouse 3T9 fibroblasts, a well-known cellular system particularly apt to the study of transcriptional changes associated with the cell cycle. Indeed, fibroblasts can be induced to quiescence by serum deprivation and then stimulated to re-enter cell cycle by serum addition ${ }^{30}$ (Fig. 1d). This fibroblast-serum model offers two key advantages to the study of target-directed miRNA degradation: (i) as changes in gene expression associated with cell cycle re-entry occur over a few hours, miRNAs dilution by cell division does not come into play (ii) data of time course analysis of gene expression and miRNA expression, transcription and decay are already available at high-resolution and at genome wide level ${ }^{27}$. When we scanned our list of target:miRNA 3C-pairs for the ones that could be studied in our model system (e.g., miRNA and target concomitantly expressed in fibroblasts), 18,856 pairs were found, with 1083 falling in the 3C-high class (Fig. 1d). We, then, stratified these pairs according to two parameters influencing the probability of a single target to promote miRNA degradation: (a) the maximum $(\max )$ change in expression of each 3C-target observed during cell cycle re-entry (Fig. 1e, $y$-axis); and (b) the max contribution (over the entire time course) of each single target to the total pool of 3C-targets of a given miRNA (\% of 3 C_Pool; Fig. 1e, $x$-axis). A few 3C-targets stood out in the analysis (Supplementary Fig. 2a), with Serpinel showing the highest dynamic range ( $\max \log _{2}$ fold-change $[\mathrm{FC}]=6.92$ ), a very high 3C-score for miR-30b-5p and -30c-5p, and a significant contribution to the 3C-target pool (of $33.8 \%$ and 35\%, respectively). 
a

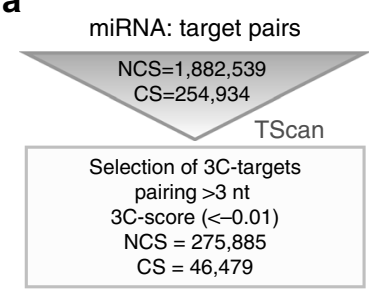

d

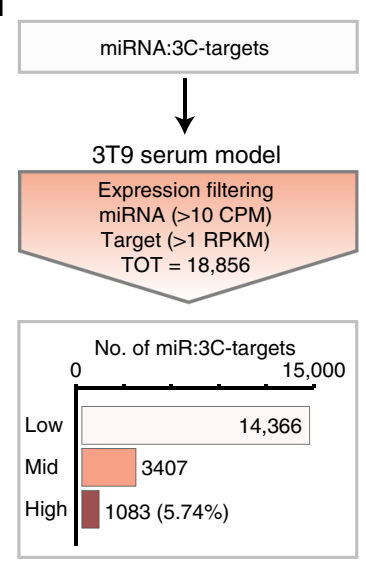

b

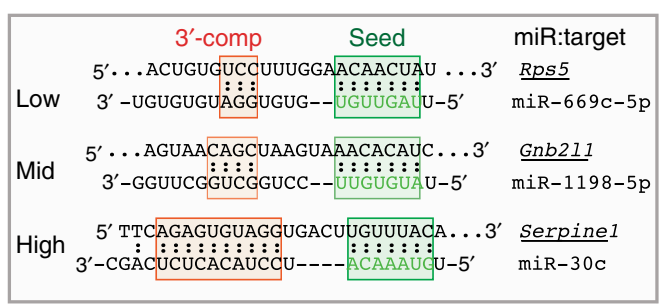

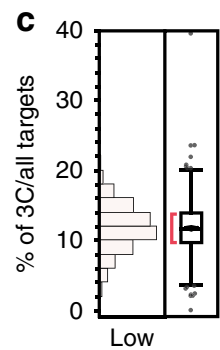

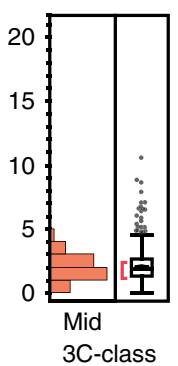

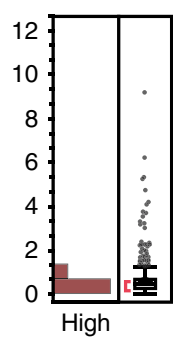
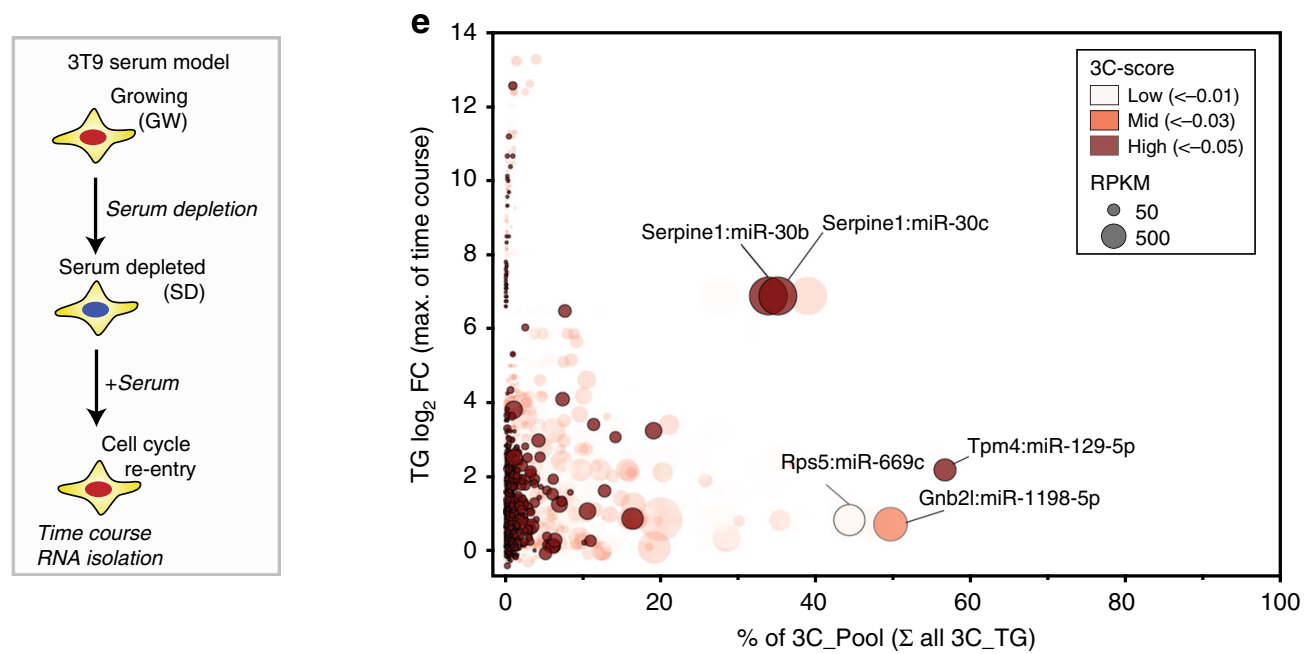

Fig. 1 Candidate TDMD targets in 3T9 fibroblasts. a Scheme summarising filtering criteria to select 3C-targets from all miRNA:target pairs present in the TargetScan6.2 database (NCS non-conserved sequence, CS conserved sequence). All 3C-targets were further classified according to their 3' complementarity score (3C-score). b Three classes of 3C-scores were identified: low, from -0.01 to -0.03 ; mid, from -0.03 to -0.05 ; and high, lower than -0.05. Representative examples are shown. $\mathbf{c}$ The fraction of 3C-targets over the total number of predicted targets, has been calculated for each miRNA and plotted as distribution, with box-plots and whiskers for each class of targets. $\mathbf{d} 3 \mathrm{C}$-targets (as in (a)) were screened to determine which ones were eligible for the 3T9 serum model (schematised on the right). Only the pairs expressed in at least one condition (growing cells, serum depleted cells or time course following serum stimulation) were retained. The distribution of filtered pairs based on the $3 \mathrm{C}$-score is also reported. e Bubble plot showing all the filtered miRNA:target pairs (from (d)) stratified according to the target max contribution (percentage; $x$-axis) to the 3C-targets pool of a miRNA $\left(\right.$ RPKM $M_{\text {target }} /$ RPKM $\left._{\text {Sum_all_3C-targets }}\right)$ and to the target max regulation $\left(\log _{2}\right.$ FC; $y$-axis) across the entire time course. Bubble size is proportional to target abundance $\left(R P K M_{\text {target }}\right)$, and target colour reflects the class of $3 \mathrm{C}$-score

Fast downregulation of miR-30b/c during cell cycle re-entry. Serpine1, also known as PAI-1, encodes a serine protease inhibitor protein that inhibits fibrinolysis as well as playing a prominent role in the early $\mathrm{G} 0>\mathrm{G} 1$ transcriptional programme $\mathrm{e}^{30}$. All five members of the miR-30 family can interact with Serpine1 transcript, as it has a high-affinity 8-mer seed-match (Fig. 2a). However, it forms an internal bulge (5 nt) and extended $3^{\prime}$ base pairings ( $9 \mathrm{nt}$ and $11 \mathrm{nt}$, respectively) only with miR-30b-5p and miR-30c-5p (herein referred to as $\mathrm{miR}-30 \mathrm{~b} / \mathrm{c}$ ) but not with the remaining members of the miR-30 family (miR-30a-5p, miR-30d$5 p$, and miR-30e-5p, henceforth referred to as miR-30a/d/e). Hence, Serpine1 is likely to act as a decay target only for miR-30b/ c. Closer inspection of the time course experiment revealed that Serpine1 expression was potently and quickly induced by serum stimulation of quiescent cells, reaching a peak at $2 \mathrm{~h}(>1000$ RPKM; Fig. 2b, left panel). Serpinel contribution to the total target pool reached its maximum at this stage, with this single transcript representing almost the entire pool of 3C-high targets for miR-30b/c ( $>90 \%$; Fig. $2 \mathrm{~b}$, right panel and Supplementary Fig. 2a). A concomitant analysis by small RNA sequencing (sRNA-seq) over the time course of stimulation, revealed that miR-30b/c levels rapidly decreased at $4 \mathrm{~h}$ after serum addition $\left(\log _{2}\right.$ FC of -0.88 and -1.13 fold-change, respectively; Fig. $2 \mathrm{c}$ ), while those of $\mathrm{miR}-30 \mathrm{a} / \mathrm{d} / \mathrm{e}$ remained almost unchanged.
Target-directed degradation has also been associated with the generation of non-templated modifications at the $3^{\prime}$ end of miRNAs (tailing and trimming isomiRs ${ }^{18,19,31}$ ). Using the IsomiRage tool to map isomiRs ${ }^{32}$, we found that $3^{\prime}$ tailing of both $\mathrm{miR}-30 \mathrm{~b} / \mathrm{c}$ had substantially increased, mirroring their downregulation patterns (Fig. 2d), while their trimming variants had been only minimally affected (Fig. 2e). Adenylation and uridylation were the prevalent modifications (Fig. $2 \mathrm{f}$ ), with about $40 \%$ of them being Aforms. The abundance of $\mathrm{miR}-30 \mathrm{a} / \mathrm{d} / \mathrm{e}$ tailed and trimmed isoforms remained substantially unchanged over the entire time course (Fig. 2d, e). We looked at the transcription of the entire miR-30 family by measuring the synthesis rate of primary transcripts during serum stimulation through a pulse labelling approach $^{33}$. It should be noted that miR-30b and $-30 \mathrm{c}$ (which has two copies, 30c-1 and 30c-2) belong to two different genetic units and, therefore, are not co-transcribed (Fig. 2g). During our time course experiment, pri-miR-30b-30d and pri-miR-30e-30c1 synthesis rates did not change considerably, while pri-miR-30a$30 \mathrm{c}-2$ transcription initially decreased and then increased slowly (Fig. 2g). No alterations were observed when looking at passenger miRNAs (Supplementary Fig. 2b). Taken together, these data suggest that the specific and quick reduction in miR-30b/c abundance during serum-induced cycle re-entry is likely the result of post-transcriptional events involving miRNA degradation. 

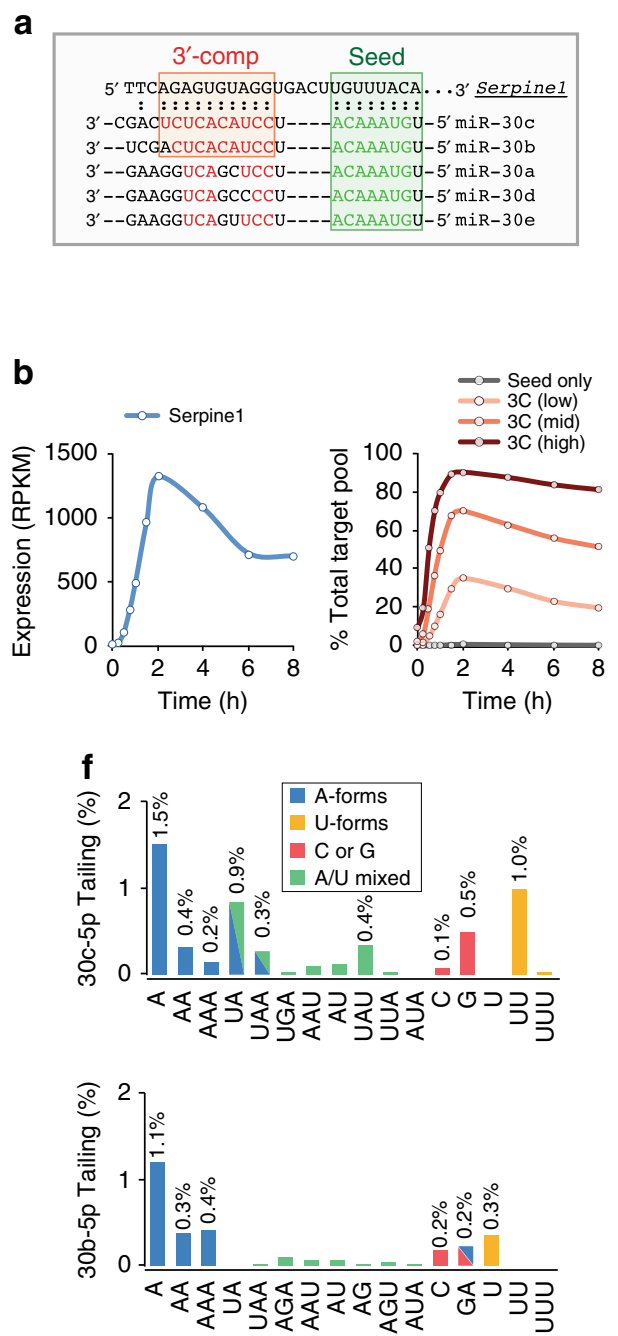
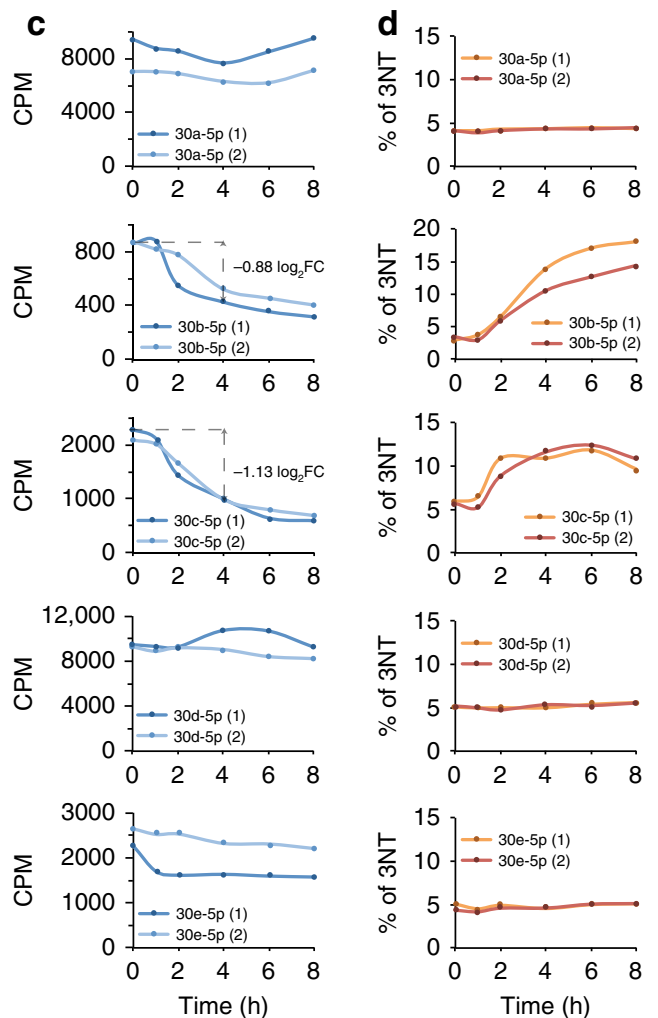

g

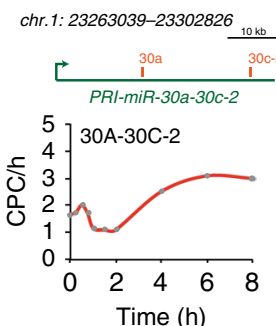

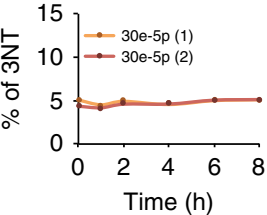

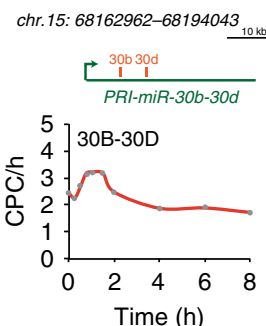

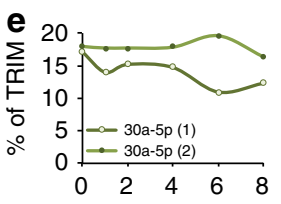
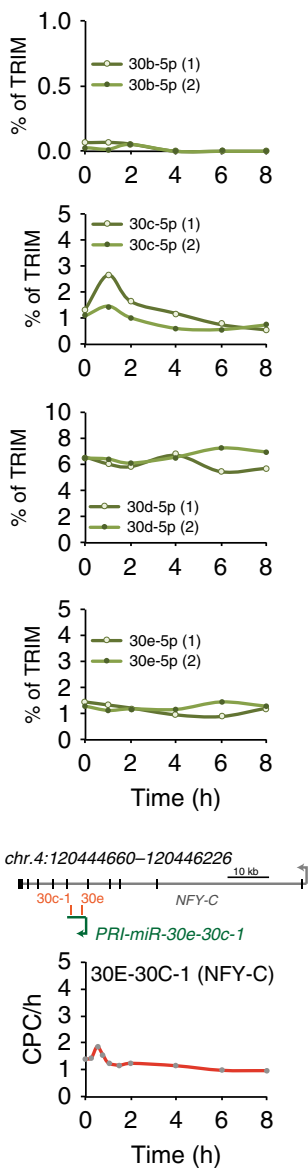

Fig. 2 Regulation of Serpine1 and miR-30 family members during serum stimulation. a Alignment of Serpine1-MRE with miR-30 family members. The seedmatch (identical for all miR-30 family members) is shown in green, and the bases of 3' complementarity, which is extensive only for two miRNAs (miR30b, 9 nt; and miR-30c, $11 \mathrm{nt}$ ), in red. b Expression of Serpine1 upon serum stimulation of quiescent 3T9 fibroblasts. Left panel, RPKM. Right panel, contribution of Serpine1 expression to the total target pool, calculated over different classes of 3C-score. c-e Expression of miR-30 family members upon serum stimulation of quiescent 3T9 fibroblasts. Shown for each miRNA are: $\mathbf{c}$ the expression level (counts per million, CPM), $\mathbf{d}$ tailing events (\% of $3^{\prime}$-nontemplated isomiRs, 3NT) and e trimming events (\% of trimmed forms, TRIM) in two independent experiments. f Frequency of tailing ( $3^{\prime} \mathrm{NT}$ isomiRs) for miR-30c-5p (top panel) or miR-30b-5p (bottom panel) in asynchronously growing 3T9 cells. Percentages of total reads. $\mathbf{g}$ Synthesis rate of the primary transcripts of the miR-30 family upon serum stimulation, as measured by a short pulse of $4 \mathrm{SU}$. Definition of genomic regions and calculation of synthesis rate as copies per cell per hour $(\mathrm{CPC} / \mathrm{h})$ have been described previously ${ }^{27}$

Serpine1 controls $\mathbf{m i R}-30 \mathrm{~b} / \mathrm{c}$ abundance in the cell. The range of Serpine1 and miR-30s expression levels in the cell was rigorously investigated by absolute quantification (copies per cell, cpc). To this aim, a titration curve was obtained using synthetic miR-30 RNA oligonucleotides and a Serpinel plasmid DNA template (Supplementary Fig. 3a-c). In exponentially growing cells (GW) miR-30c was expressed at $\sim 70 \mathrm{cpc}$ (confirmed also by dropletdigital PCR, Supplementary Fig. 3d) and miR-30b/c accounted for nearly $40 \%$ of the total miR-30 copies (Fig. 3a). In growing cells and quiescent fibroblasts, miR-30c and Serpinel were expressed at comparable levels (Serpine1: $170 \mathrm{cpc}$ in GW cells and $88 \mathrm{cpc}$ in quiescent cells; Fig. 3b), with a target-per miRNA (TPM) value of $\sim 1$ (Fig. 3c). Upon serum stimulation, Serpinel increased enormously, peaking at $>4000 \mathrm{cpc}$ (at $4 \mathrm{~h}$ ) and a $>40 \mathrm{TPM}$ ratio (Fig. 3c). Hence, Serpine1 met both molecular and quantitative requirements for eliciting $\mathrm{miR}-30 \mathrm{~b} / \mathrm{c}$ degradation. In order to directly verify the occurrence of TDMD, we devised a strategy using the CRISPR/Cas9 system $^{34}$ to manipulate Serpine1-MRE. We designed sgRNAs that would allow the deletion of a minimal region at the Serpine1 $3^{\prime}$ UTR $(\sim 160 \mathrm{nt}$, Fig. 3d), inclusive of miR30 MRE. Poorly conserved MREs for miR-224 and miR-320 were also part of the deleted region, but their corresponding miRNAs are negligibly expressed in 3T9 cells. We generated two independent mutant clones (Serpine1:miR-30 MRE-KO1 and Serpine1: miR-30 ${ }^{\mathrm{MRE}-\mathrm{KO} 2}$, hereafter referred as MRE-KO1 and MRE-KO2) (Supplementary Fig. 4a, b). In neither clones, Serpine1 mRNA and protein expression resulted affected by the manipulation of the gene $3^{\prime}$ UTR (Fig. 3e, f). In complete medium, both clones showed proliferation rates and cell cycle profiles similar to those of their parental cells (Supplementary Fig. 4c, d). When measuring global expression of miRNAs and their isoforms by sRNAseq, we noticed that, in both MRE-KO clones, only a few miRNAs were significantly induced, including miR-30c-5p (1.03 $\log _{2} \mathrm{FC}$, $p=0.0013)$ and $30 \mathrm{~b}-5 \mathrm{p}\left(0.92 \log _{2} \mathrm{FC}, p=0.0180\right)$ (Fig. 3g, 
Supplementary Fig. 4e, Supplementary Data 2). Remarkably, expression levels remained unchanged when looking either at members of the miR-30 family lacking the extended $3^{\prime}$ complementarity, or at passenger miRNAs. Quantitative reverse transcription PCR (RT-qPCR) was performed to measure primary transcripts levels for the three miR-30 loci and no difference was found between parental cells and MRE-KO clones (Fig. 3h), suggesting that the observed increase in $\mathrm{miR}-30 \mathrm{~b} / \mathrm{c}$ levels was due to a decrease in their degradation. Consistently, tailing-one of the hallmarks of target-directed degradation-of miR-30b/c decreased in MRE-KO clones, with adenylation being the most affected modification (Fig. 3i). To rule out the possibility that increased $\mathrm{miR}-30 \mathrm{~b} / \mathrm{c}$ was a by-product of clonal selection, we used RT-qPCR to measure miR-30c absolute levels in clones with a

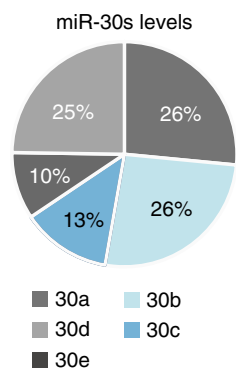

d

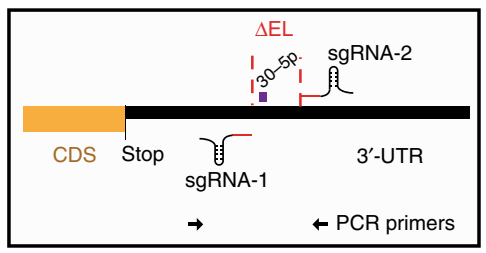

g
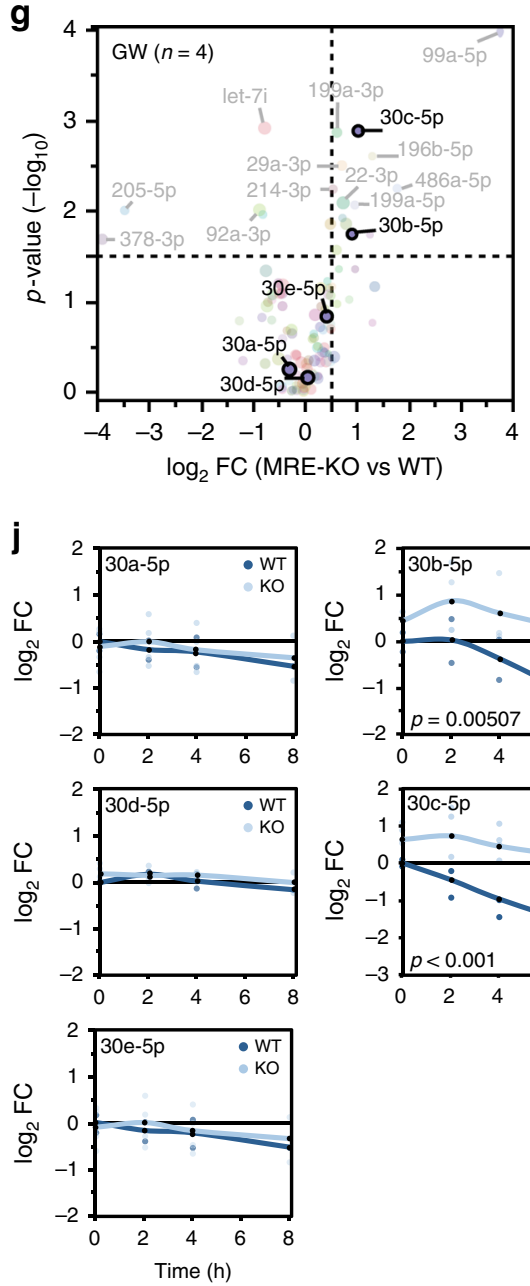

b
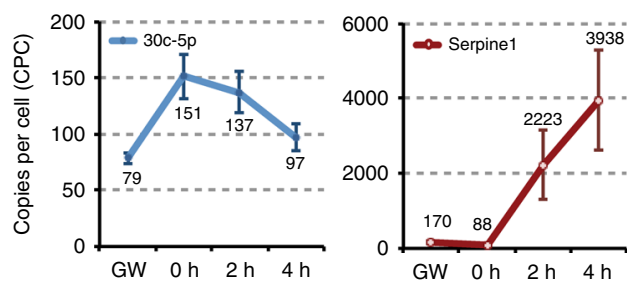

C

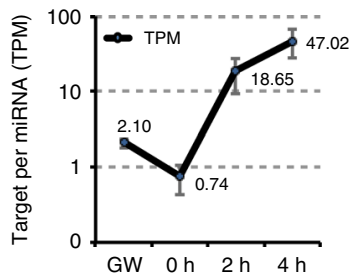

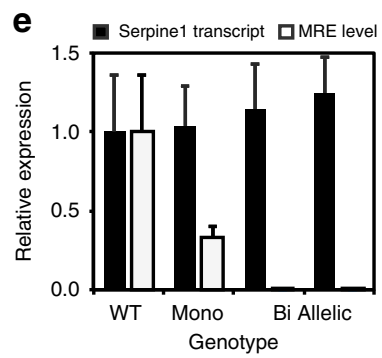

h

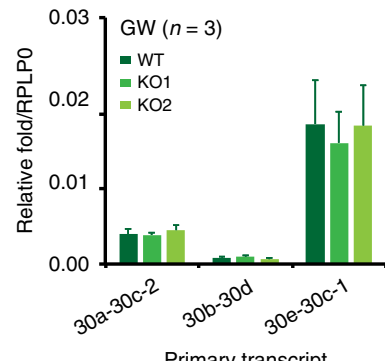

f

(KDa) WT KO2
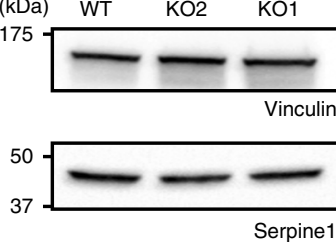
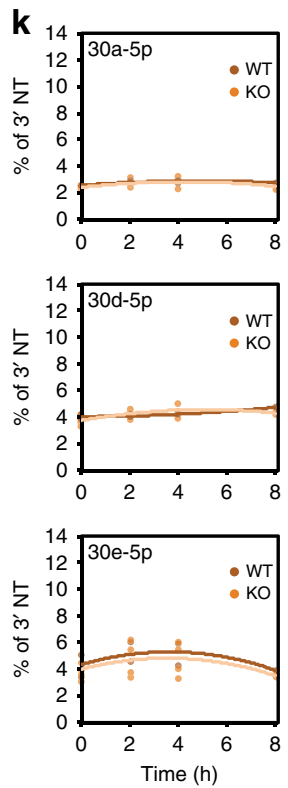
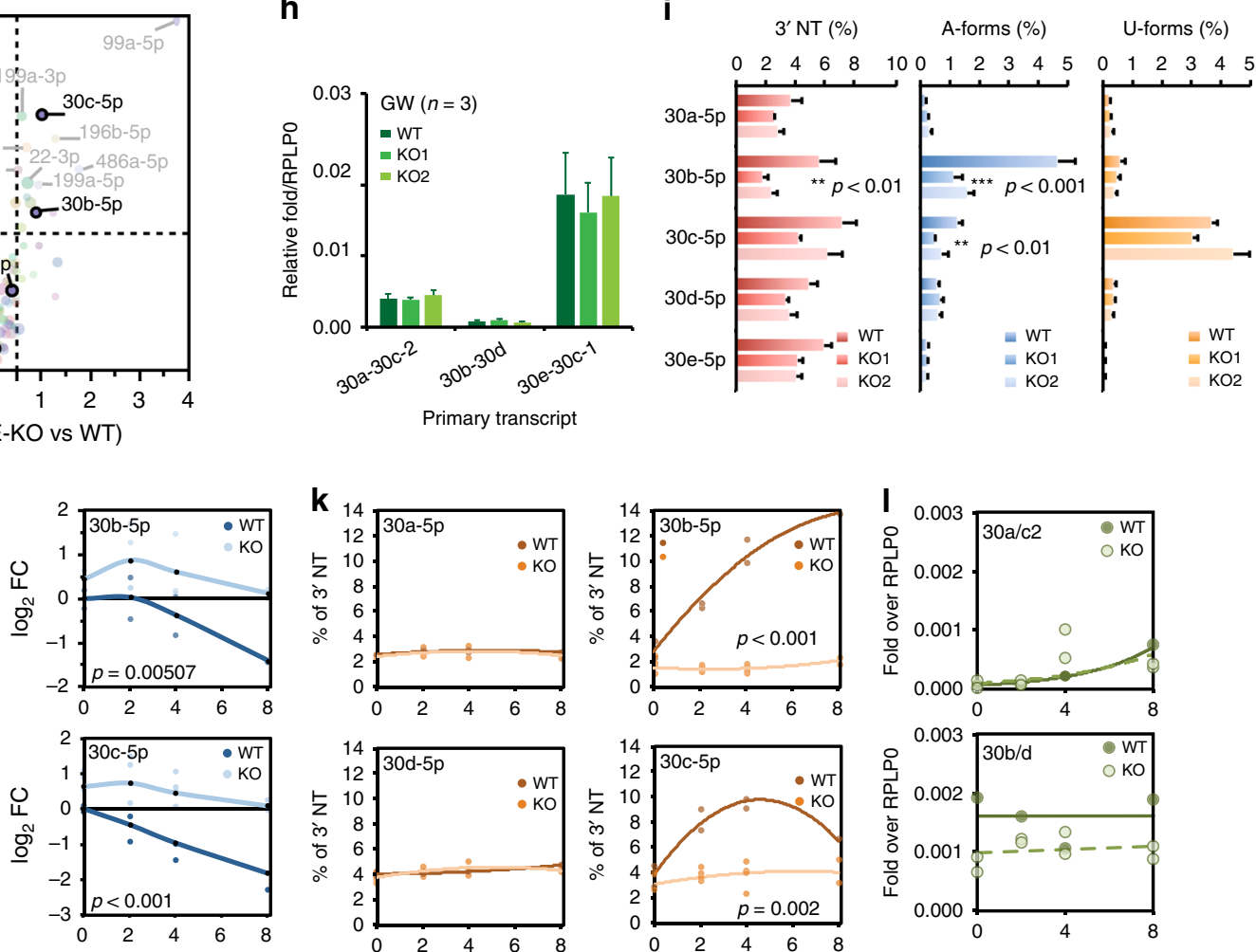

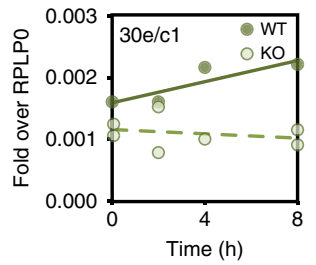


no MRE deletion (no-del clones) and found them to be the same as in their parental cells ( $\sim 80 \mathrm{cpc}$; Supplementary Fig. $4 \mathrm{f})$. In MRE-KO clones, miR-30c absolute levels instead doubled (184.7 cpc; Supplementary Fig. 4f). We also investigated miR-30 levels over the time course of serum-stimulation in MRE-KO cells. As expected, in parental cells, miR-30b/c levels were quickly downregulated upon serum stimulation (Fig. 3j), with a rapid increase of $3^{\prime}$ tailing forms (4-8 h post stimulation) (Fig. $3 \mathrm{k}$; see also Fig. 2d). In MRE-KO cells, miR-30b/c levels instead remained consistently high and no sudden increase in tailing variants was observed (Fig. 3k). While the abundance of U-forms did not change, the induction of A-forms (the large majority of tailing isoforms in parental cells) was totally abolished in MRE-KO cells (Supplementary Fig. 5b). The levels and modifications of other members of the family (i.e., 30a-d-e) were unchanged, and primary transcripts from all the three miR-30 loci stayed at similar levels over the time course (Fig. 31), further confirming a posttranscriptional regulation of $\mathrm{miR}-30 \mathrm{~b} / \mathrm{c}$ by Serpine1-MRE. In conclusion, under both steady-state (growing cells) and dynamic (serum stimulation) conditions, miR-30b/c levels are controlled by Serpine1 via a target-directed degradation mechanism.

Wild-type but not mutant MRE rescues TDMD. We next sought to rescue the interaction between miR-30 and Serpine1 3' UTR in MRE-KO cells. To this aim, we used an adenoviral construct expressing a red fluorescent tracking protein (RFP) fused to Serpine1 $3^{\prime}$ UTR (Fig. 4a). We used Serpinel wild-type sequence (SE1-WT), with its miR-30 MRE, or mutant sequences, in which the miR-30 MRE was completely replaced (SE1-MUT) or only mutated at the level of the $3^{\prime}$ complementary region, leaving the match with the miRNA seed (SE1-SEED) (Fig. 4a). First, we analysed the effects of the three constructs by RT-qPCR. The RFP-SE1-WT construct significantly reduced the levels of miR-30c and, to a lesser extent, miR-30b, without affecting any other member of miR-30 family or the unrelated miRNA let-7b (Fig. 4b). In particular, miR-30c levels decreased moderately ( 20\%) when RFP-SE1-WT construct was expressed at moderate levels $(\sim 1000 \mathrm{cpc}$, with a transduction efficiency of $\sim 30 \%$ RFP + cells) and considerably ( $\sim$-fold) when the same construct was expressed at high levels ( 10,000 cpc; nearly 100\% RFP + cells) (Fig. 4c, d). No effects were elicited by any concentration of either of the two mutant constructs (SE1-MUT and SE1-SEED), confirming that the $3^{\prime}$ complementarity is indispensable for targetdirected degradation (Fig. 4c, d). These results were fully reproduced when miRNA expression was analysed at a global level by sRNA-seq (Fig. 4e). Expression of the RFP-SE1-WT construct affected also the level of tailing variants, only in the case of miR$30 \mathrm{c}$ and miR-30b, with a marked preference for adenylation
(Fig. 4f and Supplementary Fig. 5b). Using a set of Serpine1 mutants with various levels of $3^{\prime}$ complementarity to miR-30c (Fig. 4g), we investigated $3^{\prime}$ pairing rules in the context of ectopic MRE expression. As previously observed ${ }^{18}$, four mismatches in the $3^{\prime}$ end were sufficient to nearly abolish TDMD (M4-M6 mutants, Fig. 4h), while the central bulge could be extended to up to seven nucleotides with only a modest reduction in TDMD (B7 mutant, Fig. 4h).

miR-30 activity is modulated by Serpine1. Endogenous targets involved in TDMD are also supposed to modulate the global activity of miRNAs by inducing de-repression of shared targets (Fig. 5a), similarly to ceRNAs ${ }^{13,15}$. Conversely, TDMD suppression (as in MRE-KO cells) should result in increased miRNA activity. Using a highly sensitive reporter system to measure miR30c activity at single-cell level ('miR-sensor', Supplementary Fig. 6a) we observed that repression of the synthetic target increased from 5.5-fold in parental cells to 7.7-fold in MRE-KO cells $(p<0.001$; Supplementary Fig. 6b, c). Higher levels of miR$30 \mathrm{c}$ had thus resulted in a significant increase in its activity. To gain a deeper understanding of miRNA activity at a global level and on endogenous targets, we measured the full transcriptome of parental and MRE-KO cells, in steady-state conditions (growing-GW) as well as following serum stimulation (TC) (GSE104648; Supplementary Data 3). Looking at genes that were expressed in both conditions (GW and TC, $n=9619$ ), we isolated miR-30 targets based on: (i) predictions by computational approach (TargetScan7.1: 899 conserved, CS, and 1795 non-conserved, $\left.\mathrm{NCS}^{29}\right)$; (ii) experimentally supported interactions $(n=$ 1037 for miR-30 s) obtained by High-throughput Sequencing of RNA isolated by crosslinking immunoprecipitation (HITS-CLIPfrom TarBase $\left.7.0^{35}\right)$; and (iii) miRNA:target chimaeras $(n=1037$ for miR-30c-5p), obtained by sequencing of Covalent Ligation of Endogenous Argonaute-bound RNAs (CLEAR-CLIP ${ }^{36}$, Supplementary Data 3). First, we evaluated miR-30 targets dynamics in wild-type cells. As miR-30b/c levels became strongly downregulated following serum addition (see Fig. 2c), coherently, miR30 targets were more induced (compared to background genes) at 8 and $12 \mathrm{~h}$ after serum stimulation $(p<0.0001$, Supplementary Fig. $7 \mathrm{a}-\mathrm{c})$. We, then, compared target repression in MRE-KO and wild-type cells. In growing conditions, miR-30 targets resulted slightly $\left(-0.05 \log _{2}\right.$ FC) but significantly more repressed in mutant cells (Fig. 5b, d, f). The repression was generally stronger for highaffinity MREs (8-mers and 7-mers-m8) than for low-affinity MREs (7-mers-A1) (Fig. 5b, c). Following serum stimulation, in MRE-KO cells, the downregulation of $\mathrm{miR}-30 \mathrm{~b} / \mathrm{c}$ has been shown to be abrogated (see Fig. 3j). In this dynamic setting, the repression of miR-30 targets became stronger $(p<0.0001$, Fig. 5 e, g, i),

Fig. 3 Serpine1:miR-30b/c interaction controls miR-30b/c degradation. a Percentage contribution and copies-per-cell for each member of the miR-30 family in growing cells are shown, based on absolute quantification data in Supplementary Fig. 3a-c. b Absolute quantification of miR-30c-5p and Serpine1 in 3 T9 model. Data refer to three independent biological experiments (average and s.e.m). c A target-per-miRNA (TPM) value was calculated based on absolute quantification. d Strategy for the CRISPR-Cas9-mediated removal of miR-30 MRE from Serpine13'UTR. Shown are the positions of the sgRNAs and the PCR primers used for clone sequencing (see also Supplementary Fig. 4a, b). e Expression levels of Serpine1 by RT-qPCR in wild-type (WT) 3 T9 fibroblasts and in clones with mono-allelic $(n=3)$ or bi-allelic deletion of the MRE for miR-30 (MRE-KO1 and MRE-KO2; shown individually). Different primers pairs were used to quantify total Serpine1 transcripts or just those with an intact MRE (see Methods). $\mathbf{f}$ Serpine1 protein level in WT or bi-allelic MRE-KO clones. Vinculin was used to normalise sample loading. $\mathbf{g}$ sRNA-seq was performed to measure miRNA expression in WT ( $n=4$ ) and bi-allelic MRE-KO clones (KO1 and KO2; $n=3$ each) in asynchronously growing conditions. The graph shows the average log 2 FC (MRE-KO clones as compared to WT cells; $x$-axis) and the $p$-value (- $\log _{10} ; y$-axis), by Welch's $t$-test. Colour coding reflects different miRNA families. $\mathbf{h}$ Primary transcripts for the miR-30 family measured by RT-qPCR in WT or MRE-KO clones $(n=3)$. i Total frequency (\%) of tailing events ( 3 'NT), or adenylated or uridylated only isomiRs (Aand U-forms), measured by sRNA-seq in WT $(n=4)$ or MRE-KO clones $(n=3)$; in $\mathbf{h}$, i mean and s.d., asterisks mark significant values ( ${ }^{\star \star} p<0.05$, ${ }^{\star \star \star} p<$ 0.001; Welch's t-test). j, k Expression of miR-30 family by sRNA-seq upon serum stimulation in WT and MRE-KO cells. For each miRNA, expression regulation $\left(\log _{2} \mathrm{FC} ; \mathbf{j}\right)$ and tailing events (\% $3^{\prime} \mathrm{NT} ; \mathbf{k}$ ) are shown. Details of miRNA isoforms in Supplementary Fig. 5 . I Primary transcripts of the miR-30 family by RT-qPCR. j, k, I two independent experiments shown 
a

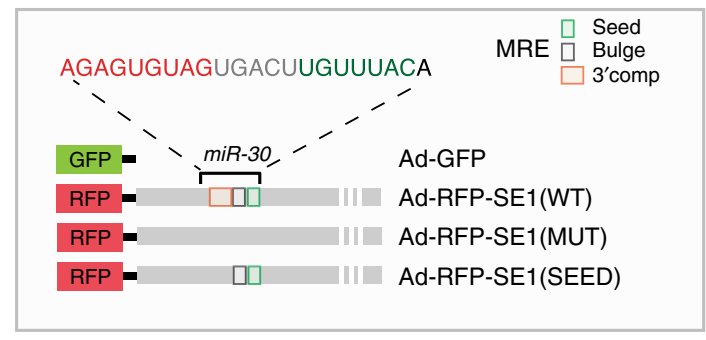

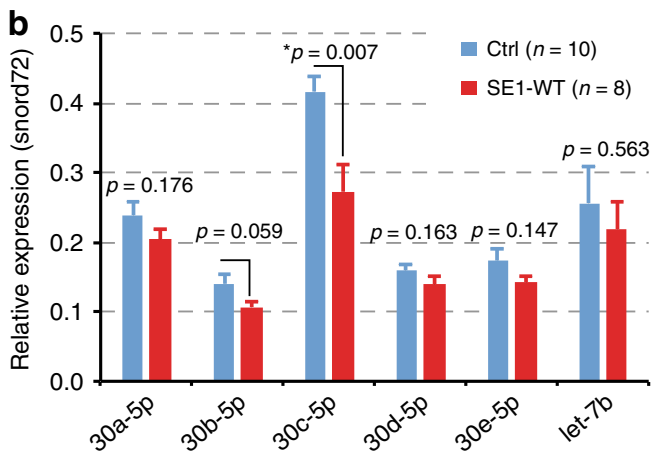
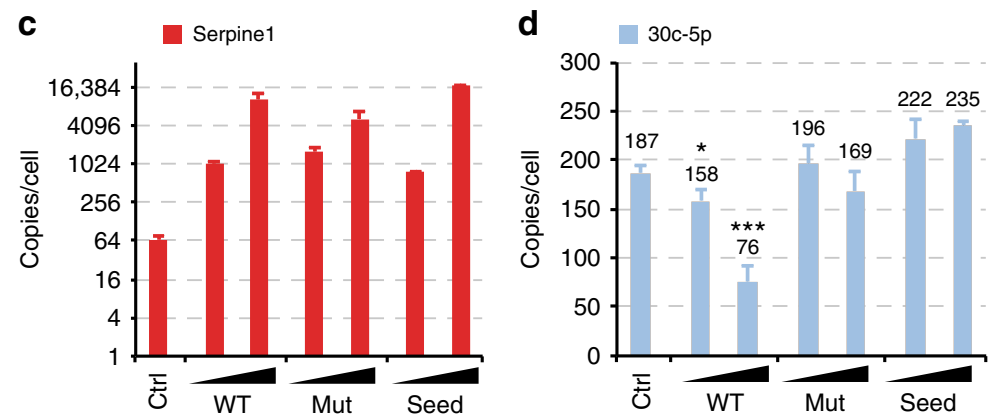

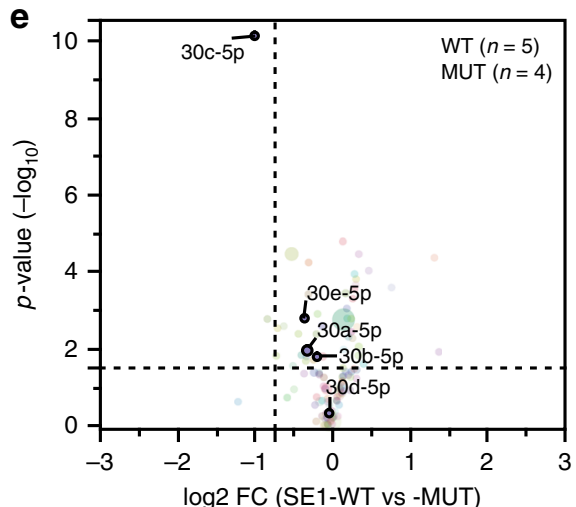

f

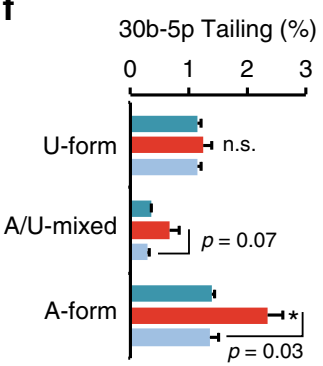

30c-5p Tailing (\%)
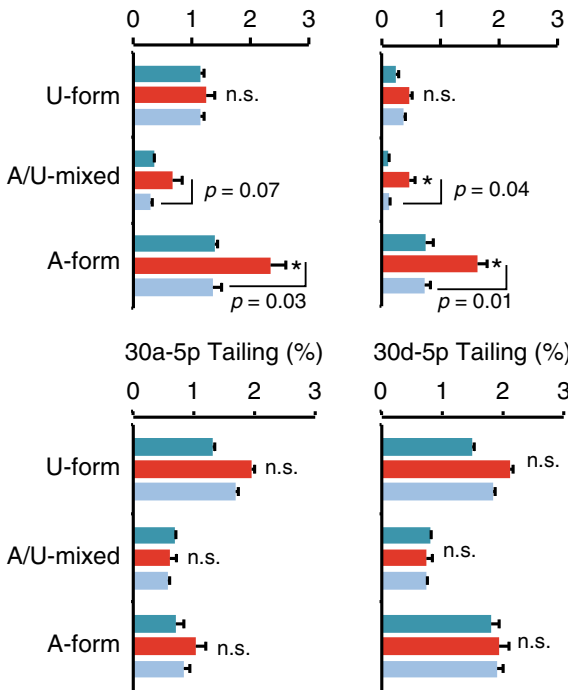

SEED $(n=3)$

WT $(n=5)$

$\operatorname{MUT}(n=4)$

30e-5p Tailing (\%)

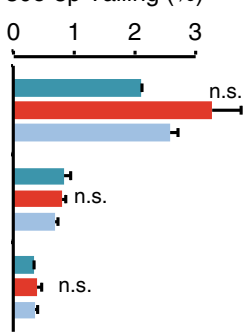

g

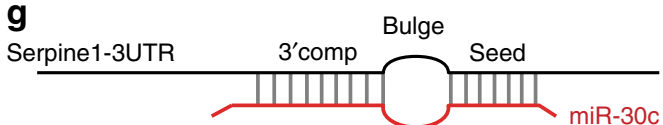

5' TCCGGAGUGUAGGUGACUUGUUUACA M4

5' TCCGCAGUGUAGGUGACUUGUUUACA M5

5' TCCGCCGUGUAGgugACUUGUUUACA M6

5' TTCAGAGUGUA ACUGACUUGUUUACA B7

5' TTCAgAguguagG UGACUUGUUUACA WT

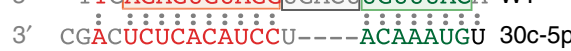

h

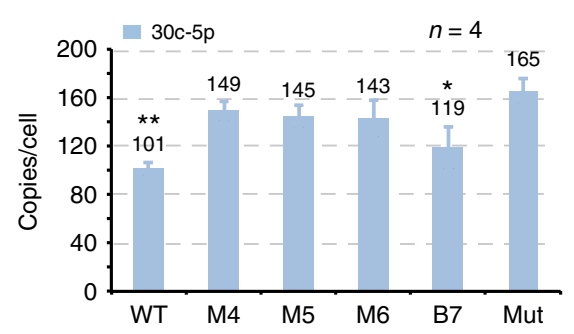

Fig. 4 Re-expression of Serpine1-MRE rescues miR-30c degradation. a Scheme of the recombinant adenoviral constructs used to re-express Serpine1 $3^{\prime}$ UTR fused to an RFP reporter (rescue experiments in (b-f)), showing the wild type form of the 3'UTR of Serpine1 (WT) and two mutants, one where miR30 MRE had been completely abrogated (MUT) and the other where the $3^{\prime}$ supplementary site had been mutated but the seed sequence left intact (SEED). b Bar plot showing the expression of miR-30 family (and Let-7b as a control) by RT-qPCR in cells infected with control virus (Ctrl) or with Ad-RFP-SE1 (WT) adenoviruses. $p$-values were determined by Welch's t-test. c, d Absolute expression (copies per cells) of Serpine1 3'UTR transcripts and miR-30c-5p measured by RT-qPCR in the rescue experiments. Adenoviruses were expressed at two different multiplicity of infection (MOI $\sim 1$ and $\sim 5$ ). Asterisks mark significant values $\left({ }^{\star} p<0.05,{ }^{\star \star \star} p<0.001\right.$; Welch's $t$-test) e sRNA-seq was performed to measure miRNA expression in MRE-KO cells that express a wild type (WT, $n=5$ ) or a mutated (MUT, $n=4)$ 3'UTR of Serpine1. The graph shows the average $\log _{2}$ FC ( $x$-axis) and the $p$-value (- $\log _{10}, y$-axis) by Welch's $t$ test. Colour coding reflects different miRNA families. f Frequency (\%) of tailing events, distinguished as adenylated (A-), uridylated (U-), or mixed isomiRs (A/U-mixed), as measured by sRNA-seq. $p$-values by Welch's $t$-test. $\mathbf{g}$ Scheme of the mutant Serpine1 constructs (see also Methods) that were used to dissect TDMD 3' pairing rules (h). Seed in green and 3' supplementary pairing in orange. $\mathbf{h}$ Absolute expression (copies per cells) of miR-30c-5p measured by RT-qPCR upon over-expression of different Serpine1 mutants (shown in (g)). Cells were collected at $36 \mathrm{~h}$ after transfection $\left({ }^{\star} p<0.05,{ }^{\star \star \star} p<0.01\right.$, Welch's $t$-test) 
with the median repression reaching $-0.2 \log _{2} \mathrm{FC}$ at both 8 and $12 \mathrm{~h}$ post stimulation (Fig. 5b, c). The specificity of the effect was confirmed by comparing the median regulation of the targets for all expressed miRNAs (Fig. 5j, $\mathrm{k}$ and Supplementary Fig. 7d): miR30 targets displayed preferential repression during serum stimulation but not in growing cells. A certain degree of specificity emerged for targets of miR-30b and -30c, as defined according to CLEAR-CLIP miRNA:target chimaeras (Fig. 5k), suggesting that miR-30b/c preferentially binds to targets with a supplementary pairing in the $3^{\prime}$ region, as previously observed ${ }^{36,37}$.

Taken together, these data strongly support a role for the Serpine1:miR-30b/c interaction in modulating the activity of a

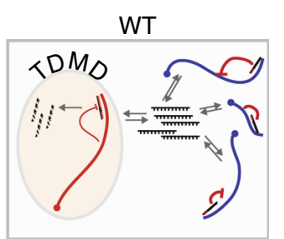

MRE-KO

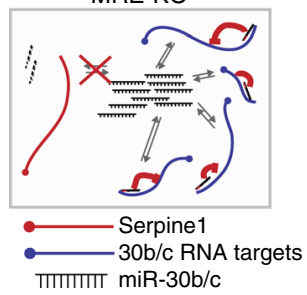

TाIIIIITा $\mathrm{miR}-30 \mathrm{~b} / \mathrm{c}$ b Target $\log _{2} \mathrm{FC}(\mathrm{KO} / \mathrm{WT})$ $\begin{array}{lll}-0.3 & 0.0 & 0.3\end{array}$

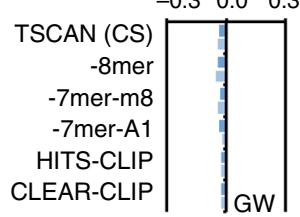

C

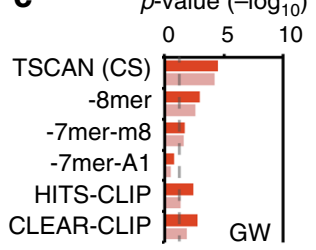

Target $\log _{2}$ FC (KO/WT)

$\begin{array}{llllllll}-0.3 & 0.0 & 0.3-0.3 & 0.0 & 0.3 & -0.3 & 0.0 & 0.3\end{array}$

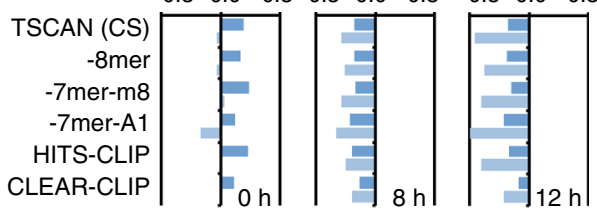

- MRE-KO1 MRE-KO2

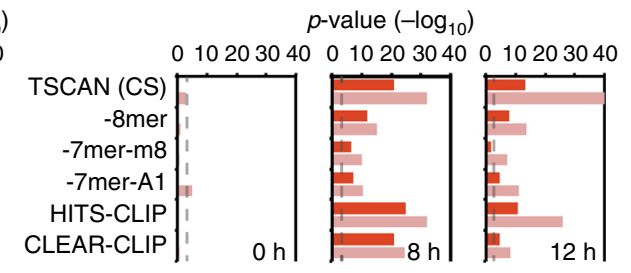

- MRE-KO1 - MRE-KO2

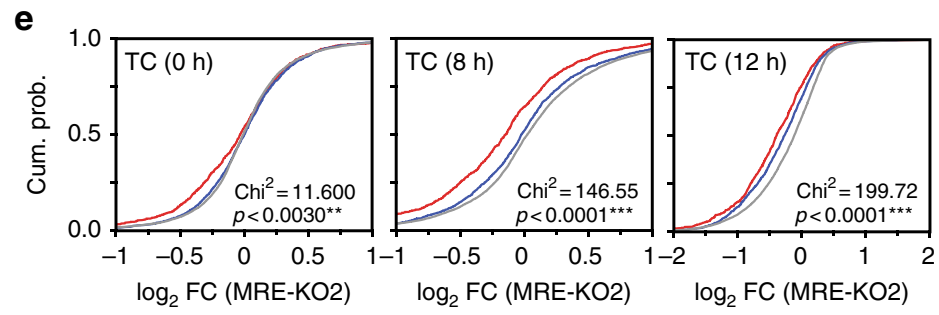

g

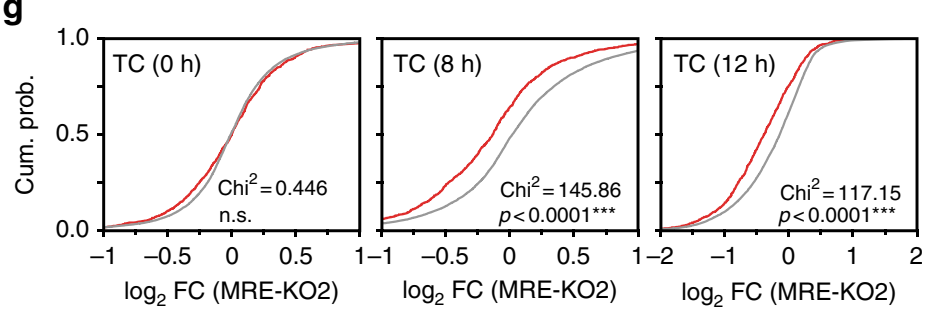

i
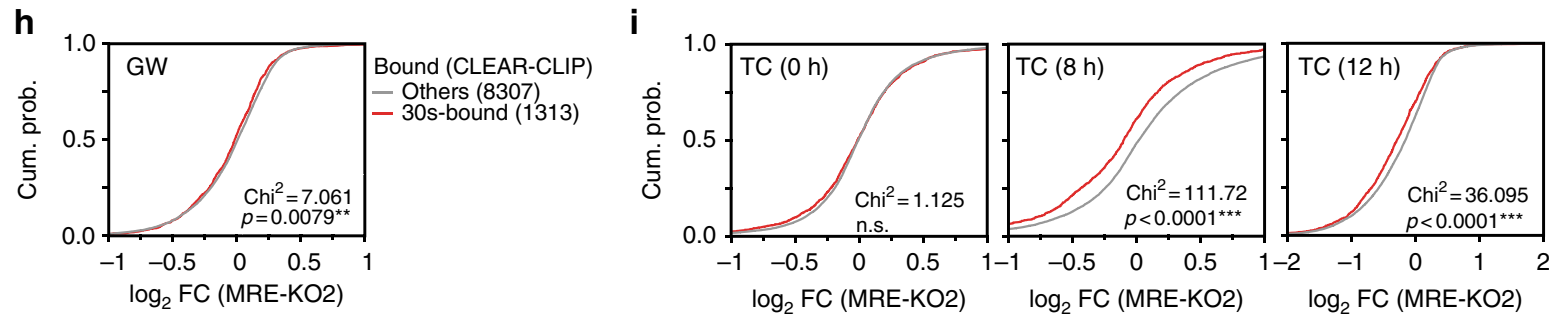

k
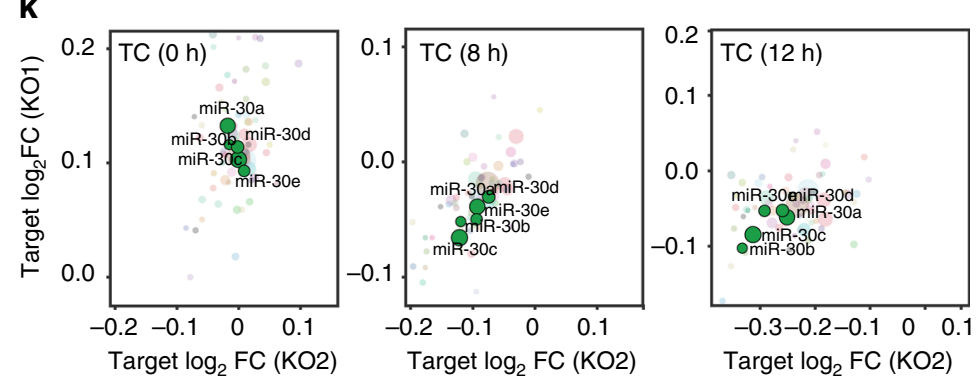
miR-30 on shared targets, particularly by temporally restricting miRNA activity during cell cycle re-entry of quiescent fibroblasts.

Serpine1:miR-30b/c interaction affects cell behaviour. We next investigated whether the increased miR-30 activity observed when the Serpine1:miR-30b/c interaction is lost, might also cause phenotypical alterations (Fig. 6a). Previous works have linked the miR-30 family to apoptosis sensitivity and p53 signalling ${ }^{38,39}$. Thus, we speculated that MRE-KO cells might be sensitised to cell death. While the basal level of apoptosis did not seem to change in growing MRE-KO cells (Fig. 6b, d), it appeared largely increased when we exposed these mutant cells to stress conditions, such as serum starvation or treatment with the anthracycline drug doxorubicin (Fig. 6c, d). Analysis of cell cycle re-entry dynamics had previously indicated that the expression levels of miR-30 targets are significantly affected in MRE-KO clones (see Fig. 5). BrdU incorporation experiments now revealed that these mutants show a remarkable acceleration in S-phase entry, (Fig. 6e, f), suggesting that G1/S transition becomes faster in the absence of a Serpine1-directed miR-30b/c degradation mechanism. When performing time-lapse microscopy analysis, we saw that MRE-KO cells had an accelerated mitotic rate (Fig. $6 \mathrm{~g}$ and Supplementary Movies 1-3), confirming that these mutants reenter cell cycle more quickly than the wild type. Notably, a significant number of MRE-KO cells also underwent apoptosis (Fig. 6h). Through gene expression analysis aimed at identifying differentially expressed genes (DEGs) in MRE-KO cells, we identified 244 DEGs in growing cells (steady-state) and 1648 DEGs in quiescent cells that had undergone serum stimulation (Fig. 7a, Supplementary Data 3 and Methods). At steady-state, we found an enrichment of genes belonging to the $\mathrm{p} 53$ pathway and the apoptotic response (Fig. 7b), which might explain the increased sensitivity to cell death of MRE-KO cells upon stress induction. No enrichment of miR-30 targets among these 244 DEGs, suggesting that the observed cellular behaviour is also due to secondary effects that originate from the disruption of the Serpine1-mediated degradation of miR-30b/c. As for the 1648 DEGs identified in stimulated cells, we used unsupervised clustering to classify them according to their temporal pattern of regulation (cluster I-V; Fig. 7c, left panel) and, based on the dynamics of serum stimulation, to distinguish constitutive (cluster I and V) from acute effects (cluster II, III, IV) (Fig. 7c, right panels). We then associated each cluster with the correspondingly enriched biological properties (Fig. 7d). In particular, compared to wt cells, MRE-KO cells showed earlier induction (cluster II) of several E2F-regulated genes involved in cell cycle and mitosis. This might explain a quicker entry into S-phase and mitosis of mutant cells (see Fig. 6e-g). Notably, clusters IIb (serum-induced genes with an earlier induction in mutant cells; e.g., Ccne2) and IV [genes repressed in mutant cells at late (8h10h-12h) time-points] were significantly enriched with miR-30 direct targets (Fig. 7e, f). In particular, cluster IV comprises miR30 targets that are key regulators of p53-pathway (Bcl6, Sirt1) and of the circadian clock (Per2, Cry2), which in turn is coupled with the cell cycle ${ }^{40}$. In summary, the Serpine1:miR-30b/c interaction profoundly affects cell behaviour by increasing sensitivity to apoptosis and by accelerating the G1/S transition during cell cycle re-entry of quiescent cells.

\section{Discussion}

Here, we provide molecular evidence that in mammalian cells a target-directed miRNA degradation mechanism exists and can control miRNA activity and cell functions. We found hundreds of targets that are potentially capable of TDMD. Focusing our study on Serpine1, we provide a proof-of-principle characterisation of TDMD at endogenous levels of expression, as opposed to acute expression of artificial targets or ectopic overexpression. Indeed, we show that under physiological conditions (i.e., both in growing fibroblasts and in quiescent cells undergoing serum stimulation) Serpine1 post-transcriptionally regulates two closely related miRNAs, miR-30b, and miR-30c, by inducing their degradation. Degradation promoted by Serpinel is characterised by some peculiar features. It involves specific post-transcriptional modifications at the miRNA $3^{\prime}$ end, with higher-than-normal levels of adenylation but no uridylation involved, as previously reported ${ }^{18}$. Finally, it only affects two members (miR-30b and 30c) of the conserved miR30 family that are not co-transcribed, thus coupling them posttranscriptionally while uncoupling them from the other three miR-30 members (miR-30a/d/e). All miR-30s can interact with Serpine1 via their seed sequence (which is shared by all miRNAs in the same family), but only miR-30b/c present an extended $3^{\prime}$ end complementarity (3C pairing) to Serpine 1 transcript. Therefore, as previously observed in vitro and with artificial targets ${ }^{18,20}, 3 \mathrm{C}$ pairing is essential in the case of a TDMD mechanism triggered by endogenous targets. Accordingly, Serpine1 has stronger effects on miR-30c than on miR$30 \mathrm{~b}$, as miR-30c forms additional $3^{\prime}$ base pairings with Serpine1. The importance of $3 \mathrm{C}$ pairing is highlighted also by the fact that it is required in reconstitution experiments. Additional mismatches can be tolerated close to the bulge, but completely impair miRNA degradation if located in proximity of miRNA 3' end. Complementarity is not the only requisite for TDMD, which also presents some context-dependent quantitative requirements. Therefore, we performed absolute measurements of miRNA and target expression (copies per cells) and computed the corresponding target-per miRNA value (TPM) to investigate the stoichiometry of their interaction. Upon serum stimulation of quiescent cells, Serpine1 is strongly induced so to

Fig. 5 miR-30 activity increases in Serpine1-MRE-KO cells. a Scheme representing WT and MRE-KO cells expected behaviour in terms of miR-30c activity with canonical targets (in blue) and decay targets (in red). When TDMD is suppressed (i.e., by removing the MRE from a TDMD target, like Serpine1), miRNA increases in abundance and is redistributed on targets thus increasing its repression activity. $\mathbf{b}, \mathbf{c}$ Expression analysis of WT and MRE-KO cells was performed by RNA-seq in different conditions of cell growth (GW: growing cells; 0 h: quiescent cells; $8 \mathrm{~h}$ and $12 \mathrm{~h}$ : serum-stimulated cells). b Bar charts report the difference between the median log2 fold changes (MRE vs WT) of different subsets of miR-30 targets. The analysis was performed with targets either predicted by TargetScan7.1 (897 conserved targets, CS, also broken down by seed-match as 8-mer, 7-mer-m8, or 7-mer-A1) or validated by HITSCLIP (1037 targets, from Tarbase v7.0 ${ }^{35}$ ) and CLEAR-CLIP (1315 targets ${ }^{36}$ ). c $p$-values of data shown in (b). d, e Cumulative distribution functions (CDF) of $\log _{2}$ FC of mRNA (MRE vs WT) for conserved targets (CS), not conserved targets (NCS) and genes not predicted as targets (Other). $\mathbf{f}, \mathbf{g}$ CDF plot as in (d, e) with miR-30 targets from HITS-CLIP experiments ${ }^{35}$. h, i CDF plot as in (d, e) with miR-30 targets from CLEAR-CLIP experiments ${ }^{36}$. j, $\mathbf{k}$ Targets for all expressed miRNAs were identified by CLEAR-CLIP. The bubble plots report the median log2 fold changes (MRE-KO vs WT), calculated for every miRNA having at least 50 expressed targets. Colour code is for miRNA families. Members of the miR-30 family are highlighted in green. All statistical analyses (chi-square and $p$-value) were performed using the Wilcoxon test 
a

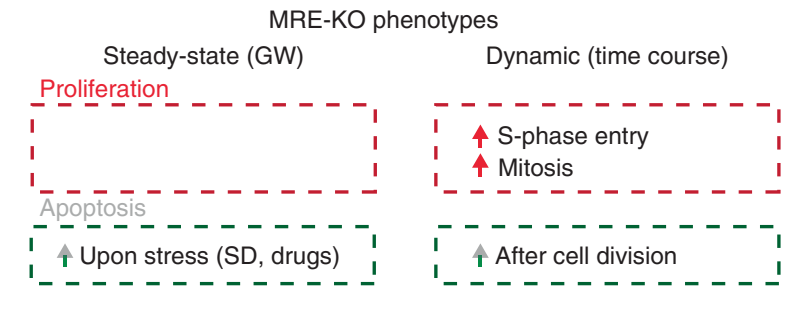

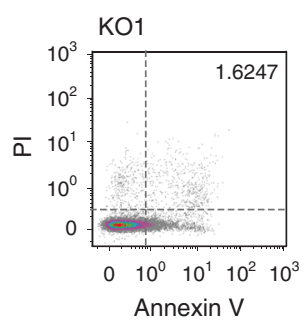

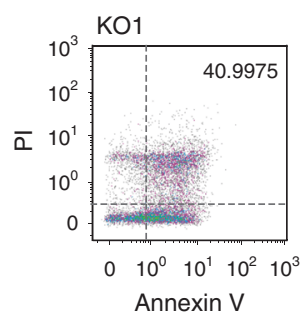

C
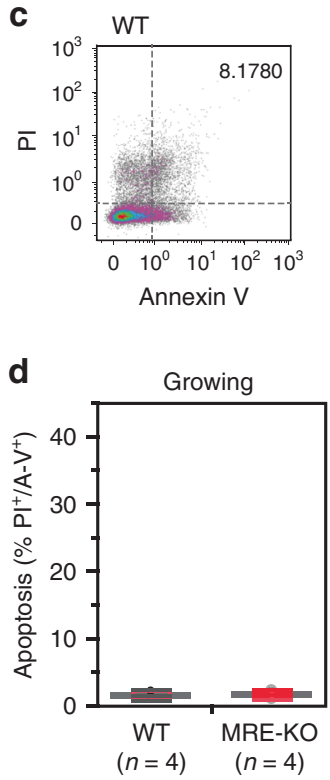

b

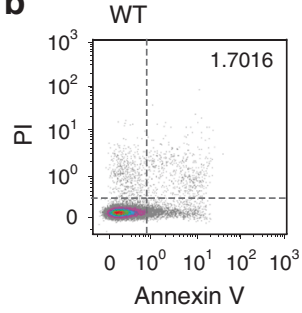

Serten

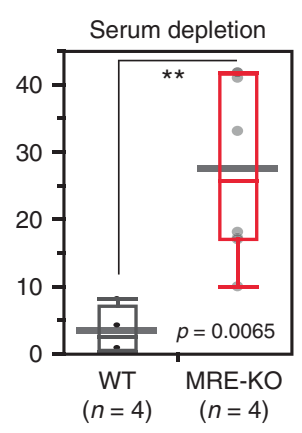

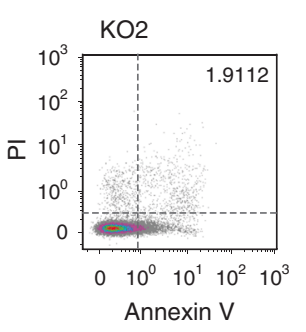
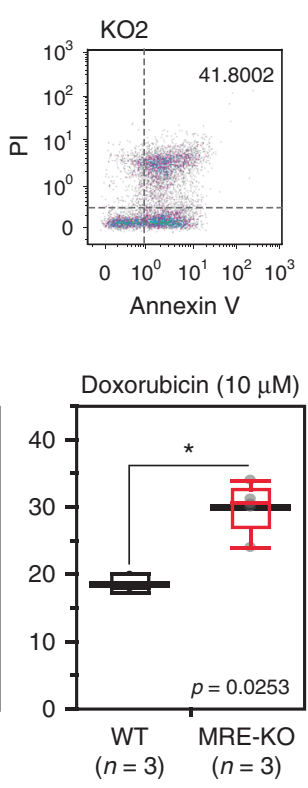

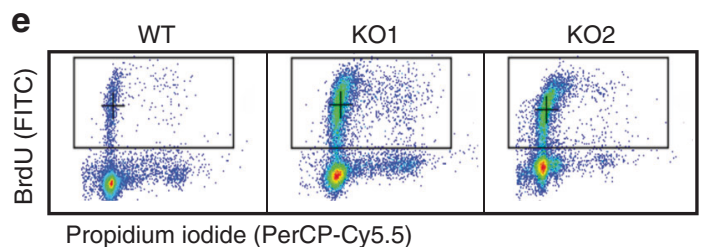

f
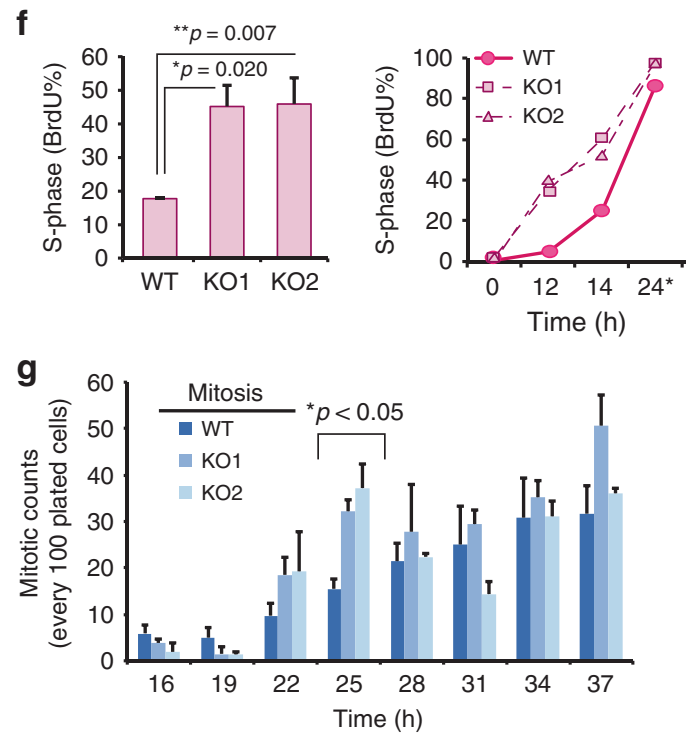

$\mathbf{h}$

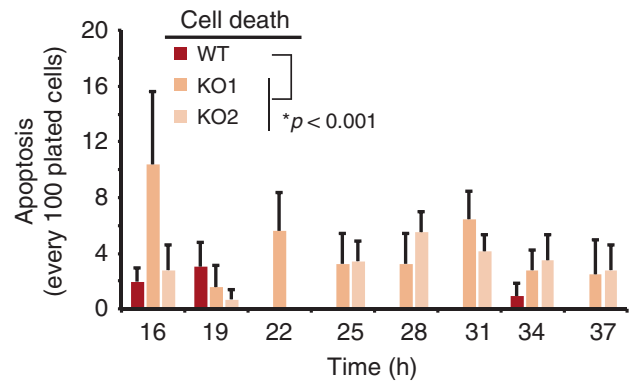

Fig. 6 MRE-KO cells display an altered cell cycle re-entry and are sensitised to apoptosis. a Summary of the phenotypes of MRE-KO cells. b, c Sensitivity to apoptosis was analysed in MRE-KO clones and WT cells by FACS profiling; apoptotic cells were identified by propidium iodide (PI) and Annexin-V staining; b asynchronously growing condition; c cells were serum depleted for $48 \mathrm{~h}$. $\mathbf{d}$ Box-plots of apoptotic cells $\left(\mathrm{Pl}^{+}\right.$and Annexin- $\left.\mathrm{V}^{+}\right)$in WT and MRE-KO clones in different conditions. Asterisks mark significant values $\left({ }^{\star} p<0.05,{ }^{\star \star} p<0.01\right.$, Wilcoxon test). e Dynamics of cell cycle re-entry induced by serum stimulation by FACS analysis. S-phase entry was determined by BrdU incorporation (30 min pulse) and propidium iodide staining. $\mathbf{f}$ Left: S-phase entry evaluated at $14 \mathrm{~h}$ post-serum stimulation (average and s.e.m.; $n=3$ ). Right: line chart shows a single time course of cell cycle re-entry ( $24^{\star}$ : cells treated in continuous with BrdU for $12 \mathrm{~h}$ ). $\mathbf{g}$, h Number of mitosis (g) and cell deaths (h), normalised for every 100 plated cells, occurring between 16 and $37 \mathrm{~h}$ after serum stimulation as analysed by time-lapse microscopy $(n=3)$. $\mathbf{f}, \mathbf{g}, \mathbf{h} p$-value by Welch's test

largely exceed the levels of $\mathrm{miR}-30 \mathrm{~b} / \mathrm{c}(\mathrm{TPM}>10)$ and very rapidly halve their levels (i.e., half-life of $\sim 4 \mathrm{~h}$ ). No changes in transcription or processing are concomitantly observed (see Fig. 2). This effect is completely dependent on Serpine1, as $\mathrm{miR}-30 \mathrm{~b} / \mathrm{c}$ repression is abolished in MRE-KO cells. Thus, a sudden and massive upregulation of a TDMD target can have a role in accelerating miRNA degradation within a precise time window. Indeed, we had previously estimated a longer half-life for miR-30b and $-30 \mathrm{c}(\sim 12$ and $16 \mathrm{~h}$, respectively) in growing fibroblasts ${ }^{27}$. In growing cells Serpine1 is expressed at relatively low levels $(170 \mathrm{cpc})$ and in range with. the number of miRNA copies (about $70-80 \mathrm{cpc}$ for miR-30c and 140 for miR-30b). Nevertheless, miR-30b and miR-30c are subjected to TDMD also in growing cells, as in MRE-KO cells the levels of the two
miRNAs almost doubled. Therefore, endogenous TDMD takes place even for targets that are present at relatively low levels of expression, provided that they are in a stoichiometric range with their cognate miRNAs $(\mathrm{TPM} \geq 1)$. The reason being that every time the miRNA interacts with the target, it is irreversibly subtracted from the miRNA pool by degradation ${ }^{16}$. It is worth mentioning that in reconstitution experiments, where targets are provided ectopically, TDMD requirements differ slightly as Serpine1-MRE acts mostly on miR-30c-5p and, in order to do so, must reach higher levels of expression (at least $\sim 1000 \mathrm{cpc}$ ). In these experiments, miRNA levels were analysed at $36 \mathrm{~h}$ post infection. It is conceivable that a longer time interval is necessary to observe a significant reduction in miR-30b abundance, as this is expressed at relatively higher levels than 


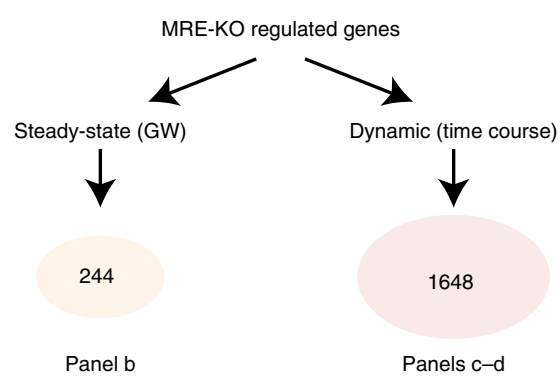

c

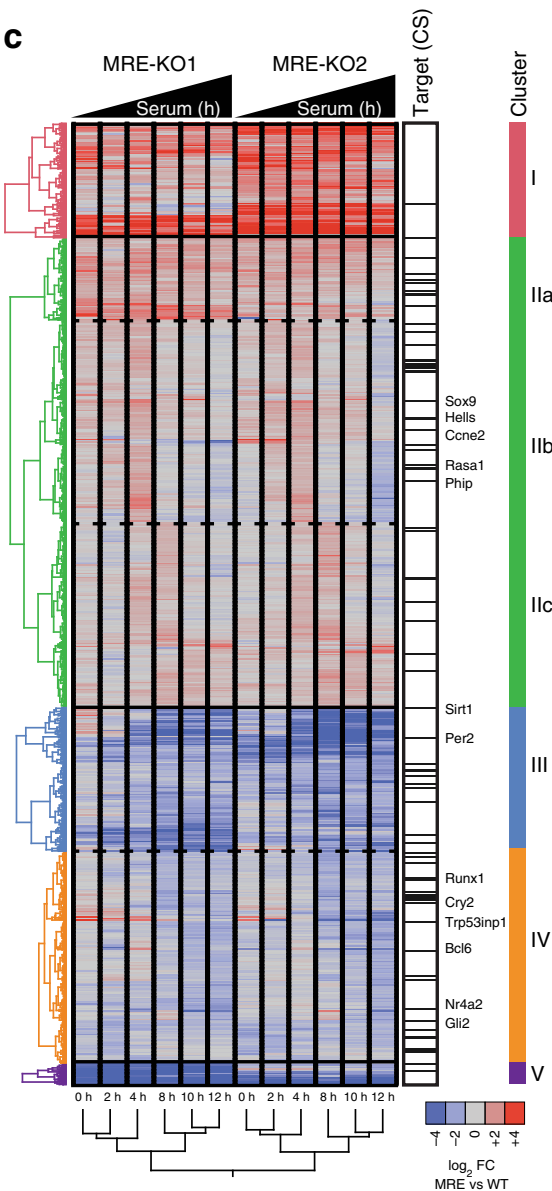

e

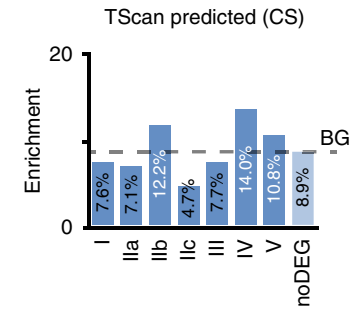

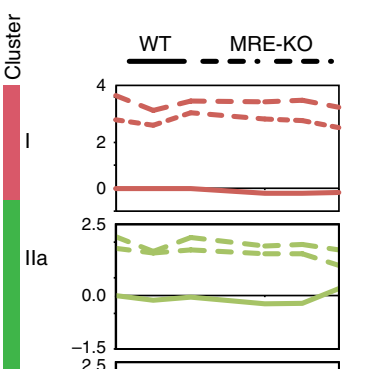

$\mathrm{llb}$
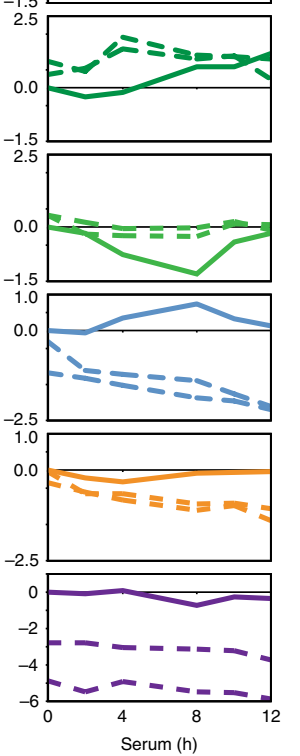

b Upregulated genes

REACTOME_CHONDROITIN_SULFATE_METABOLISM REACTOME METABOLISM OF CARBOHYDRATES NABA_MATRISOME

REACTOME_GLYCOSAMINOGLYCAN_METABOLISM

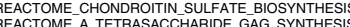
NABA_BASEMENT_MEMBRANES

\section{Downregulated genes}

HALLMARK P53 PATHWAY

KEGG_APOPTOS̄IS

SA CASPASE CASCADE

PID CASPASE PATHWAY

BIOCARTA_MITOCHONDRIA_PATHWAY

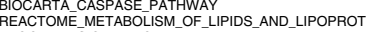

REACTOME_METABOLISM_OF_LIPIDS_AND_LIPOPROT
KEGG P53_IIGNALINGPATWWA
HALLMARK_EPITHELIAL_MESENCHYMAL_TRANSITION

d

Constitutive upregulated (high)

NABA_MATRISOME

NABA_CORE_MATRISOME

NABA_ECM_G LYCOPROTEINS

REAC TOME EXTRACELLULAR MATRIX ORGANIZA

Constitutive upregulated (low)

HALLMARK_EPITHELIAL_MESENCHYMAL_TRANSITION

NABA_MATRISOME

FEATOME_EXTRACELIULAR MATRIX_ORGANIZATION

Serum-induced upregulated

HALLMARK_MITOTIC_SPINDLE

ALLLARK_E2F_TARGETS

REACTOME_KINESINS

REACTOME CELL CYCLE MITOTIC

Serum-repressed upregulated

HALLMARK_E2F_TARGETS

KEGG_AMINOACYL_TRNA_BIOSYNTHESIS

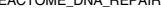

REACTOME_G2_M_CHECKPOINTS

EACTOME_CELL_CYCLE

DEACTOME FANCONI ANEMIA PATHWAY

REACTOME_CELL_CYCLE_MITOTIC

Serum-induced downregulated

NABA_MATRISOME

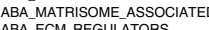

NABA ECM AFFILIATED

NABA_ECM_AFFLLATED
REACTOME_EXTRACELLULAR_MATRIX_ORGANIZATION
NABA_CORE_MATRISOME

Serum-repressed downregulated

HALLMARK_TNFA_SIGNALING_VIA_NFKB

HALLMARK_ADIPOGENESIS

HALLMARKADSPATHWAY

KEGG CIRCADIAN RHYTHM MAMMAL

HALLMARK KRAS SIGNALING UP

HALLMARK_KRAS_SIGNALING_DN

KEGG_PATHWAYS_IN_CANCER

促

HALLMARK HYPOXIA

Constitutive downregulated (high) NO ENRICHMENT

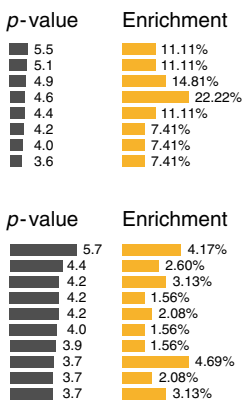

$p$-value Enrichment

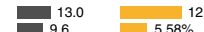

\begin{tabular}{l}
$5.58 \%$ \\
$5.58 \%$ \\
\hline
\end{tabular}

\begin{tabular}{l}
$5.58 \%$ \\
$-4.06 \%$ \\
\hline $7.11 \%$
\end{tabular}

$7.11 \%$
$-3.05 \%$

$2.03 \%$

p-value Enrichment

- $5.8 \quad 4.86 \%$

$\begin{array}{ll}5.3 & 9.22 \\ 5.0 & 4.26 \%\end{array}$

p-value Enrichment

\begin{tabular}{ll}
17.0 & $5.51 \%$ \\
\hline & 15.7
\end{tabular}

$\begin{aligned} & 15.7-5.22 \% \\ & 10.7\end{aligned}-232 \%$

$2.32 \%$
$3.77 \%$

$3.77 \%$
$1.45 \%$
$-3.77 \%$

$1.45 \%$
$3.77 \%$
$3.19 \%$

p-value Enrichment

$7.5-3.46 \%$

$3.46 \%$
$-1.89 \%$

$2.20 \%$

$1.87 \%$
$1.57 \%$
$3.77 \%$

$3.77 \%$
$-1.57 \%$

$1.26 \%$

p-value Enrichment

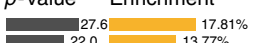

\begin{tabular}{c}
$12.02 .0 \quad 6.07 \%$ \\
\hline
\end{tabular}

$\begin{array}{ll}6.7 & 3.64 \% \\ 6.6 & 2.83 \%\end{array}$

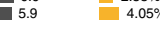

$p$-value Enrichment

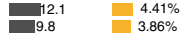

$3.86 \%$
$3.31 \%$

$3.31 \%$
$4.68 \%$
$-1.10 \%$

$1.10 \%$
$2.48 \%$

$2.48 \%$
$-38 \%$
$-3.03 \%$

$2.48 \%$
$-3.03 \%$
$-2.20 \%$

$2.20 \%$

\section{f miR-30s bound (CLEAR-CLIP)}
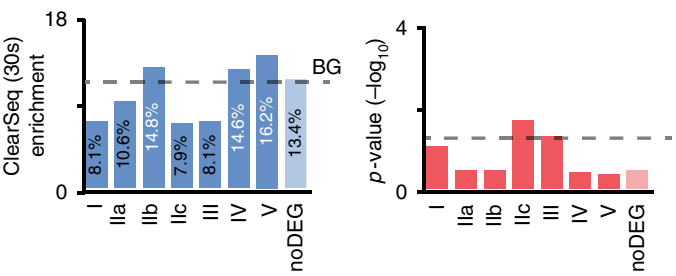

Fig. 7 MRE-KO cells display altered gene expression. a Differentially expressed genes (DEGs) between WT and MRE-KO clones were determined by RNAseq either at steady-state (growing cells, $n=244$ ) or upon serum stimulation $(n=1648)$ of quiescent cells. $\mathbf{b}$ Enriched biological functions among GWDEGs were retrieved by Gene Set Enrichment Analysis (GSEA), calculating the overlap with curated gene sets (Molecular Signatures Database; shown those with a $p$-value $<0.001)$. c Unsupervised hierarchical clustering of serum stimulation DEGs (1648 genes) identifies five major clusters (I-V) showing different patterns of gene regulation. Cluster II was further split into three sub-clusters (Ila-b-c) having different behaviour in terms of serum-regulation dynamics. MiR-30 family conserved targets predicted by TargetScan are also reported. Right charts: median gene regulation in each (sub)cluster is reported over the time course; MRE-KO data were normalised over basal expression level (i.e., quiescent state, $0 \mathrm{~h}$ ) of WT cells. $\mathbf{d}$ Enriched biological functions among clusters shown in $\mathbf{b}$. e, $\mathbf{f}$ Enrichment (and chi-square, $p$-value by Contingency Test) of miR-30 targets across each cluster was calculated either using predictions (e) or validated interactions (f) 
miR-30c. It should be also stressed that, at low MOI, some cells were uninfected and did not ectopically express Serpine1-MRE (as determined by monitoring RFP). This might explain why ectopically expressed Serpine1 triggered TDMD at higher levels (at least $\sim 1000 \mathrm{cpc}$ ) when compared to endogenously expressed Serpine1 $(170 \mathrm{cpc})$.

How many endogenous TDMD targets do exist? We chose to study Serpine1 as it was one of the top hits in a list of candidate targets displaying ideal structural characteristics: a high-affinity seed (8-mer or 7-mer-m8), a central bulge ( $>2$ and $<5$ nts) and a high 3C-score (high degree of complementarity at the $3^{\prime}$ end). Based on our preliminary observations, we can anticipate that at least 1000 target:miRNA pairs (e.g., $0.05 \%$ of all predicted targets) present molecular characteristics that make them suitable for TDMD. While this work was under revision, a different case of endogenous TDMD was provided by Bitetti and co-workers ${ }^{17}$. In their study, the NREP transcript promotes degradation of miR-29b in neuronal cells. The NREP:miR-29b and Serpine1:miR-30b/c pairs share the same complementarity features. Significantly, NREP is almost exclusively expressed in the brain (where TDMD is very effective) and imparts a spatial restriction to miR-29b expression in the cerebellum. Loss of the NREP:miR-29b interaction generates an in vivo phenotype characterised by impaired motor functions in fish and mice. A relevant role for TDMD in animal behaviour has thus been claimed. However, the impact of NREP on shared miR-29 targets was not investigated. Endogenous targets involved in TDMD are expected to influence miRNA-mediated repression. By investigating the Serpine1:miR-30 case, we provide compelling evidence in favour of such hypothesis. MRE-KO cells show a significant increase in miR-30 activity, as confirmed using both a synthetic reporter directly monitoring miRNA activity (miR-sensor) and endogenous targets identified by prediction algorithms or experimental approaches. In growing conditions (at steady-state), effects on endogenous targets are mild (with a 5\% increase in repression) and no major changes in phenotype are observed. Gene expression alterations observed in this context are likely to be a consequence of clone adaptation to the new (increased) levels of miR-30b/c. On the contrary, MRE-KO cells show remarkable phenotypes when exposed to acute stresses. In particular, upon serum stimulation mutant cells fail to properly regulate $\mathrm{miR}-30 \mathrm{~b} / \mathrm{c}$ levels, enter more quickly into S-phase and display an increased mitotic and cell death rate. In this context, strong $(\sim 20 \%)$ and specific repression of miR-30 targets is observed, with a certain degree of specificity towards $\mathrm{miR}-30 \mathrm{~b} / \mathrm{c}$ preferential targets (as opposed to shared miR-30 targets). Indeed, two independent studies mapping miRNA:target interactions suggest that miR$30 \mathrm{~b} / \mathrm{c}$ preferentially bind those targets that possess supplementary pairing in the $3^{\prime}$ region $^{36,37}$. In future, it would be worth trying to identify such specific targets and characterise them in serum-stimulated cells (e.g., by CLASH), as they might help clarify miR-30b/c function in cell cycle re-entry. In conclusion, we have provided evidence that in mammalian cells endogenous targets can control miRNA levels posttranscriptionally and thus modulate miRNA activity on all their targets.

We can no longer consider miRNA targets as being all equal. The way has now been opened to the identification of new endogenous TDMD targets that might be involved in different physiological or pathological processes. TDMD confers a further level of flexibility to miRNA regulation. It can restrict miRNA expression to a specific subset of cells (regulation in space-as for NREP:miR-29b in the cerebellum ${ }^{17}$ ) or impart temporalrestriction to miRNA activity in dynamic settings (regulation in time-as for Serpine1:miR-30b/c during cell cycle re-entry).

\section{Methods}

Statistical analysis. Analyses (Oneway, Scatter Plot, Contingency) and statistics were produced using JMP 12 (SAS) software. Microsoft Excel was used to generate bar graphs with average and standard deviation (s.d.) or standard error mean (s.e. m.) of repeated experiments. The number of replicates and the statistical tests used are indicated in the figure legends.

Cell treatments and procedures. Mouse 3T9 fibroblasts a kind gift of Bruno Amati. Cells were cultured in DMEM (Dulbecco's modified Eagle's medium) supplemented with $10 \%$ fetal bovine serum (FBS), $100 \mathrm{U} / \mathrm{ml}$ penicillin and $100 \mathrm{mg} /$ $\mathrm{ml}$ streptomycin. Cells were routinely checked for mycoplasma contamination and resulted negative. 3T9 cells were induced to quiescence in a serum-deprived medium $(0.1 \%$ FBS ) for 3 days. Cell cycle re-entry was stimulated by adding fresh medium with $10 \%$ FBS. For cell cycle analysis, cells were treated with $33 \mu \mathrm{M} 5$ bromo-2'-deoxyuridine (BrdU) for $30 \mathrm{~min}$, harvested by trypsinisation, and ethanol-fixed. Staining was performed with anti-BrdU primary antibody (1:5 in PBS 1\% BSA, BD Biosciences Cat. 347580) and anti-mouse FITC-conjugated secondary antibody (1:50 in PBS 1\% BSA, Jackson Immunoresearch Cat. 115095-075). DNA was stained with overnight incubation with $2.5 \mu \mathrm{g} / \mathrm{ml}$ propidium iodide (Sigma). Samples were acquired on a FACS-CALIBUR (BD Biosciences) flow cytometer and analysed using FlowJo 10 software. Live time-lapse analysis of mitosis and apoptosis during cell cycle re-entry was performed with a high-content screening station (ProScan II, Prior) equipped with a microscope incubation chamber, imaging cells in bright field with a $20 \times /$ NA 0.45 objective (Nikon Eclipse TE2000-E inverted microscope) every 15 min starting at $12 \mathrm{~h}$ after serum stimulation and reconstructed with ImageJ software. For each sample, mitosis and cell deaths were manually annotated at each time frame: $\sim 200$ cells were followed (in three independent replicas) and data reported as number of mitosis (or cell deaths) occurring every 100 cells.

Apoptosis analysis by Annexin-V-PI staining. Cells were grown in normal conditions or under starvation, and treated or not with $1 \mu \mathrm{M}$ of doxorubicin (Sigma) for $48 \mathrm{~h}$ to induce apoptosis. After treatment, adherent as well as detached cells were harvested and labelled with Annexin-V-APC (1:50 in annexin buffer, eBioscience Cat. BMS306APC) for $45 \mathrm{~min}$. Next, cells were labelled with propidium iodide (PI, Sigma) and immediately analysed by MACSQuant Flow cytometer (Miltenyi). Data analysis was performed by FlowJo software (TreeStar).

Cell viability analysis. Cells were plated in 96-well plate and analysed at 24, 48, 72 and $96 \mathrm{~h}$ by Cell Titre GLO (Promega) following the manufacturer's protocol.

Isolation of 4sU-RNA. Starting from $40 \mu \mathrm{g}$ of total RNA diluted in $100 \mu \mathrm{l}$ of RNase-free water, $100 \mu \mathrm{l}$ of biotinylation buffer $(2.5 \times$ stock: $25 \mathrm{mM}$ Tris $\mathrm{pH} 7.4$, $2.5 \mathrm{mM}$ EDTA) and $50 \mu \mathrm{l}$ of EZ-link biotin-HPDP $(1 \mathrm{mg} / \mathrm{ml}$ in DMF; Pierce/ Thermo Scientific 21341) were added and incubated for $3 \mathrm{~h}$ at room temperature (RT). Unbound biotin-HPDP was removed by adding chloroform/isoamyl alcohol (24:1) and purifying the mix using MaXtract high-density tubes (Qiagen). RNA was precipitated by adding $\mathrm{NaCl}(5 \mathrm{M})$ at $1 / 10$ volume and isopropanol at equal volume. Biotinylated RNA was resuspended in water $\left(55^{\circ} \mathrm{C}, 5^{\prime}\right)$, quantified with a Nanodrop 1000 spectrophotometer (to keep track of the yield after the first phase of the procedure) and then purified using $50 \mu \mathrm{l}$ of Dynabeads MyOne Streptavidin T1 (Invitrogen). Before mixing with RNA, beads were washed twice in wash buffer A $(100 \mathrm{mM} \mathrm{NaOH}, 50 \mathrm{mM} \mathrm{NaCl})$ and once in wash buffer $\mathrm{B}(100 \mathrm{mM} \mathrm{NaCl})$ and then resuspended in $100 \mu \mathrm{l}$ of buffer $\mathrm{C}(2 \mathrm{M} \mathrm{NaCl}, 10 \mathrm{mM}$ Tris pH 7.5, $1 \mathrm{mM}$ EDTA, $0.1 \%$ Tween-20) to a final concentration of $5 \mu \mathrm{g} / \mu \mathrm{l}$. RNA was added in an equal volume and rotated at RT for $15 \mathrm{~min}$. Beads were washed three times with wash buffer D ( $1 \mathrm{M} \mathrm{NaCl}, 5 \mathrm{mM}$ Tris pH 7.5, $0.5 \mathrm{mM}$ EDTA, 0.05\% Tween-20). RNA was eluted from the beads in $100 \mu \mathrm{l}$ of $10 \mathrm{mM}$ EDTA in $95 \%$ formamide $\left(65^{\circ}\right.$ C, $10 \mathrm{~min}$ ). The eluted fraction was diluted in TRIzol ${ }^{\circledR}$ LS Reagent (Life Technologies; cat. no. 10296-028), and RNA was extracted with the miRNeasy Micro Kit (Qiagen; cat.no. 217084) and then eluted in $14 \mu \mathrm{l}$ of RNase-free water. $4 \mathrm{sU}$ RNA quantification was conducted with Qubit ${ }^{\circledR}$ (Life Technologies).

Small RNA sequencing and data analysis. Total RNA, including small species, was isolated through the miRNeasy Mini Kit (Qiagen). When the expected yield was $<1 \mu \mathrm{g}$, the miRNeasy Micro Kit (Qiagen) was used. Small RNA sequencing (sRNA-seq) libraries were prepared using $1000 \mathrm{ng}$ of total RNA with the Illumina TruSeq $^{\text {TM }}$ Small RNA Kit, following the manufacturer's instructions. Sequencing was performed on an Illumina HiSeq 2000 at 50 bp single-read mode and 20 million read depth $(8 \times)$. Sequencing quality was checked in the FASTQC report, considering only experiments with Q30 or above (Phred Quality Score). Analysis was performed with the IsomiRage workflow, as previously described ${ }^{32}$. Raw data together with detailed description of the procedures are available in GEO database (GSE104650). Normalisation was performed with the library size (reads-per-million) and then (only for the serum stimulation experiments) further corrected for serum-induced RNA amplification as previously reported ${ }^{26}$. 
RNA sequencing and data analysis. Total RNA was extracted with the miRNeasy Mini (Qiagen) and then 1000 ng were purified with Ribozero rRNA removal kit (Illumina). Libraries were generated with the TruSeq RNA Library Prep Kit v2 (Illumina). Next, sequencing was performed on an Illumina HiSeq 2000 at $50 \mathrm{bp}$ single-read mode and 50 million read depth $(3 \times)$. Reads were aligned to the $\mathrm{mm} 9$ mouse reference genome using the TopHat aligner (version 2.0.6) with default parameters. Differentially expressed genes (244 DEGs shown in Fig. 7a, b) were identified using the Bioconductor package DESeq2 based on read counts, considering genes whose $q$ value relative to the control was lower than 0.05 and whose maximum expression was higher than RPKM of 1 . For the identification of genes differentially expressed in MRE-KO cells upon serum stimulation (1648 genes; Fig. 7a, c-f), we compared MRE-KO cells with parental cells at each time-point and we considered all the expressed genes $(\mathrm{RPKM}>1)$ with a $\log 2 \mathrm{FC}>|1|$ in both mutant clones (MRE-KO1 and MRE-KO2) at least at one time-point of the time course. For miRNA-target analysis (shown in Fig. 5), mouse miR-30 targets were downloaded from TargetScan 7.1, including information on conservation and on the type of seed (8-mers, 7-mer-m8, and 7-met-1A). HITS-CLIP-validated miR-30 targets were downloaded from TarBase $7.0^{35}$. CLEAR-CLIP targets were previously published ${ }^{36}$ : in particular we focused on the mouse data set, containing brain target:miRNA interactions and we filtered only those interactions occurring in $3^{\prime}$ UTR region.

miRNA, pri-miRNA and mRNA RT-qPCR expression analyses. Mature miRNA detection by RT-qPCR was performed with miScript system (Qiagen). In particular, miR-30c-5p and miR-30b-5p were detected using miScript Primer Assay MS00001386 and MS00011725. Primary transcripts of miRNAs (pri-miRNAs) were evaluated by quantitative PCR on total RNA. RT-qPCR was performed using SuperScript ${ }^{\circledR}$ VILO cDNA Synthesis Kit (Life Technologies cat.no. 11754050) and Fast SYBR green master mix (Life Technologies). About $25 \mathrm{ng}$ of cDNA were used as the input to detect pri-miRNAs, and $5 \mathrm{ng}$ to detect mRNAs. Primer pairs were designed using computer-assisted primer design software (Primer3), preferentially in the $500 \mathrm{bp}$ upstream of the sequence of the mature miRNA according to mm10 genome. The complete list of RT-qPCR primers used in this study is in Supplementary Table 1.

miRNA expression analyses by droplet-digital PCR. Absolute quantification of miR-30c was performed by droplet-digital PCR on a QX200 system (Bio-Rad), following the manufacturers' instructions. Briefly, $10 \mathrm{ng}$ of RNA was reversetranscribed with miRCURY LNA Universal RT microRNA PCR kit (Exiqon). Then, ddPCR reactions were prepared replacing Exiqon PCR master mix with QX200 EvaGreen ddPCR Supermix (Bio-Rad) and diluting 1:4 Exiqon PCR primer mix for miR-30c-5p (cat. 204783). Serial dilutions of cDNA (0.4/0.2/0.1/0.05 ng) were used as to measure assay linearity. Droplets were generated and PCR amplification performed following EvaGreen cycling conditions. Finally, we read the droplets on a QX200 droplet reader. We typically obtained $\sim 20,000$ droplets for each sample.

miRNA 3C-target analysis. Mouse miRNA targets were downloaded from TargetScan 6.2 (i.e., the latest version that includes $3^{\prime}$ pairing contribution scores in downloadable data files). 3C-targets were selected according to criteria outlined in Fig. 1a. Both the conserved sites (CS) and non-conserved sites (NCS) were used. Targets were then classified according to the $3^{\prime}$ end pairing contribution, a feature that measures the presence of additional binding regions (in addition to the seed region) located at the $3^{\prime}$ end of the miRNA. Those targets containing $3^{\prime}$ end supplementary regions were referred to as '3C-target genes' (3C_TG) and classified in three classes (HIGH, MID, LOW) according to the $3^{\prime}$ pairing contribution score, as shown in Fig. 1e. Targets expressed in 3T9 mouse fibroblasts were identified by crossing the list of 3C_TGs with RNA-seq data sets (growing cells and serumstimulated cells). Genes with FPKM values $<1$ were considered as 'not expressed' and, therefore, excluded. Finally, we selected targets of expressed miRNAs in 3T9 cells, by considering sRNA-seq data and filtering miRNA $>10 \mathrm{cpm}$.

Generation of Serpine1-MRE30c deletion mutant. sgRNAs flanking the miR-30 MRE were designed for ENST00000223095.4 (Serpinel transcript), using the CRISPR Design Tool ${ }^{34}$. SgRNAs were subsequently cloned in PX458 vector and sequence-verified. 3T9 fibroblasts were co-transfected with two PX458 vectors (sgRNA_1 and sgRNA_2) harbouring single sgRNAs sequence (5 $\mu \mathrm{g}$ of DNA for each construct). At $24 \mathrm{~h}$ and $48 \mathrm{~h}$ post-transfection, cells were harvested for FACS sorting. The top $2 \%-3 \%$ of GFP + cells were sorted as individual cells into 96 -well plates. After 2 weeks, 96-well plates were duplicated and deletions in clonal cells were screened by PCR (Supplementary Fig. 4a) using specific primer to detect mono-allelic or bi-allelic deletions. Positive clones were confirmed by DNA sequencing (Supplementary Fig. 4b). The protocol efficiency was about $20 \%$ for mono-allelic and $2 \%-5 \%$ for bi-allelic deletions. PCR primers for clone screening were Serpine1_crisp_FW: GGAGGGCACAACACTTTCAT; and Serpine1_crisp RW: AGTGCTTCTTCTCCCCAACA. MRE-KO clones used in this work were produced by the following sgRNAs:

sgRNA_1(-) Sense: caccgAAGCAAGCTGTGTCAAGGGA

Antisense: aaacTCCCTTGACACAGCTTGCTTc
sgRNA_2 (+) Sense: caccgTCTCCCAGTGGGGGGGCCCT Antisense: aaacAGGGCCCCCCACTGGGAGAc

Note that the efficient transcription from the $\mathrm{U} 6^{-}$promoter requires a $5^{\prime} \mathrm{g}$.

Serpinel protein expression in MRE-KO clones was analysed by western blot (PAI-1 monoclonal antibody, 1D5, dilution 1:1000, Thermo Fisher Cat. MA517171).

Rescue of MRE expression by adenoviral constructs. In order to re-express miR-30 MRE, recombinant adenoviruses were produced using the AdEasy System (Agilent), following manufacturers' instructions. Briefly, gene synthesis (GenScript) generated a mCherry-Serpine1 (3'UTR) construct (see below for the complete sequence) that was cloned into pShuttle vector (Agilent) by adding KpnI-NotI sites at the extremities. After homologous recombination in BJ5183-AD-1 cells (Agilent), positive recombinants (isolated by $\mathrm{PacI}$ digestion) were used to produce adenoviruses in 239Ad cells. Viruses were finally concentrated with Adeno-X maxi purification kit (Takara).

mCherry-Serpine 3'UTR construct (underlined with a solid line the miR-30 MRE):$$
5^{\prime}-
$$

GGTACCCGCCACCATGGTGAGCAAGGGCGAGGAGGATAACATGGCCAT CATCAAGGAGTTCATGCGCTTCAAGGTGCACATGGAGGGCTCCGTGAAC GGCCACGAGTTCGAGATCGAGGGCGAGGGCGAGGGCCGCCCCTACGAG GGCACCCAGACCGCCAAGCTGAAGGTGACCAAGGGTGGCCCCCTGCCCT TCGCCTGGGACATCCTGTCCCCTCAGTTCATGTACGGCTCCAAGGCCTAC GTGAAGCACCCCGCCGACATCCCCGACTACTTGAAGCTGTCCTTCCCCG AGGGCTTCAAGTGGGAGCGCGTGATGAACTTCGAGGACGGCGGCGTGG TGACCGTGACCCAGGACTCCTCCCTGCAGGACGGCGAGTTCATCTACAA GGTGAAGCTGCGCGGCACCAACTTCCCCTCCGACGGCCCCGTAATGCAG AAGAAGACCATGGGCTGGGAGGCCTCCTCCGAGCGGATGTACCCCGAG GACGGCGCCCTGAAGGGCGAGATCAAGCAGAGGCTGAAGCTGAAGGAC GGCGGCCACTACGACGCTGAGGTCAAGACCACCTACAAGGCCAAGAAG CCCGTGCAGCTGCCCGGCGCCTACAACGTCAACATCAAGTTGGACATCA CCTCCCACAACGAGGACTACACCATCGTGGAACAGTACGAACGCGCCGA GGGCCGCCACTCCACCGGCGGCATGGACGAGCTGTACAAGTAGCTCGAG CAGTGGGAAGAGACGCCTTCATTTGGGACGAAACTGGAGATGTTATAAG CAGAAACTCTGAAGAAAAGGATTATTTAAAGGACTCTATGGGGAGAAAG AGAAGGCAACTCCTCCTTACCCCCCACACTGGTAATCTTTCCAACCAGCA TCCCAGACCTCGGACTCTTGAAGGGAAAAGAGTCTAACTCCCTCCTCCC TAGGGATTCCTACCCCACAAAGGTCTCATGGACCATAGAACTCACAGTA CCTGGATCTGCCCAGCATGCCCTTTGGACCCAGTTCCCACCGAGGCCCC AGCAGAGTGGAGGGCACAACACTTTCATTCAGCAAAATCGTTTGTGTTC CAGTCACACTGTGGGCACCTCTTGCATCGCCTGCCATTGCTGTGGAGGG TGGCCATGGGCCAAAGGAAAAAGCACTGTCCTATCTCAAGGTCCACTGT GGAAATGTCCACCTTGCCCACCTCCAAGGGGCAACGGATAGACAGATCA AATGGTGGCCCAATAGCGAGCCTTCTCCCTGCTCCCTCCCTTGACACAGC TTGCTTATGTTATTTCACGCGTAGAGTGTAGGTGACTTGTTTACAGAGC TCCAGCTTTTTTCGACCCACAAACTTTTTTCATTTGGAAAGGGTGTAAGA AAAGTCGGACGTGTGTGTGCCTGGCTCTTCGTCCCCAGTCTCCCAGTGG GGGGGCCCTGGGGAGATTCCAGGGGTGTGATTGAATATTTATCTCTTGC TCTTGTATGTTTGTTGGGGAGAAGAAGCACTTTTAAGGAAAATGCTTCT TATTTAAACCGTGGCATACGGCATCCCATTTGGGGTCTGCATCCCTGTAT GTCAGGGGTGCATCACTCCACAAACCTGCCCCTCTGGGTAGCCTCGTGA TGGGGCTCACACTGCCGCCTAGTGGCAGCCGAACACACCCTTACCCGGT CССТСССТСССТССССССССССССССССССССCCCGTGGCTCTTTTCСТT AGGGACCTTGCCAAGGTGATGCTTGGCAACCCACGTTAAAGGAAGGGGG GAAAAAAGATTAGATGGAAGAGAGAGAGATTTGAGAGAGGGCAAAGTG GTTTCAAATTTTTCCAAGGCATTCAGAAGCAGAGAGGGAAAAGGGGCTG TGTGACCTAACAGGACAGAACTTTCTCCAATTACTGGGTGAGTCAGAGC TGCACTGGTGACTCACTTCAATGTGTCATTTCCGGCTGCTGTATGTGAG CAGTGGACACGTGGGGGGGCGGGGGGGGGATGAAAGAGACAGCAGCTC CTGGTCAACCACCTTAGTTAGATAATCTTTTTTGAAAGCTTCCTAGCTGG AGGTATGATCAGAAAACCAATTTACTGAAAAACTGCACAAGAAGGTACG GTGAATGTAATTTCCTAGCAGGCCACTCTGCATCTGTTATGTCTCCACCG GAAAAAAAATAATCATGTTGGTGTTTTTGCTTTTCTCTCTCTCCСTCTTTC TCTCTGATTTTTTTTTCCTCTCTTTTCATTATGCACTGGACAGCCACACAC CGTGTACCCATAGGGCCCCAAATGTGGGGTCACATGGTCTTGAATTTTG TTGGTTACATATGCCTTTTTGTTGTTGTTTGTCTTCACTTTTGATATATAA ACAGGTAAATATGTTTTTTAAAAAATACTAAATATAGAGAATATGCAAA CAAAAGCGGCCGC-3

GGTACC KpnI

ACGCGT $M l u I$

GAGCTC SacI

GCGGCCGC Not 1

In order to produce other adenoviral constructs, the following oligonucleotides were subcloned into MluI-SacI sites flanking the miR-30 MRE:

Ad-RFP-SE1(MUT)

FW: cgcgtACTCTGAAAGTGAAAAGCCAATGgagct

REV: cCATTGGCTTTTCACTTTCAGAGTa

Ad-RFP-SE1(SEED)

FW: cgcgtGATATTGTCCTGACTTGTTTACAgagct

REV: cTGTAAACAAGTCAGGACAATATCa 
Expression of mutant-binding sites for miR-30. In order to re-express different variants of Serpine1 ${ }^{\mathrm{miR}-30 \_M R E}$ in MRE-KO cells, we cloned MRE $+\sim 20$ surrounding bases (Supplementary Table 2) into EcoRI-XhoI sites that were put after the stop codon of a eGFP cloned in a pcDNA3.1 $(+)$ mammalian expression vector (Thermo Fisher Scientific).

Vectors were transfected in MRE-KO cells with Lipofectamine 3000 reagent (Thermo Fisher Scientific). Cells ( $>75 \%$ GFP positive by FACS analysis) were collected for analysis after $48 \mathrm{~h}$.

Measurement of miR-30 activity (miR-sensor) by flow cytometry. Sequences harbouring four complementary repeats for miR-30c-5p or a control sequence (see below) were purchased from TwinHelix and cloned (XbaI/XmaI) into the $3^{\prime} \mathrm{UTR}$ of a destabilised GFP (dGFP) in a bidirectional lentiviral vector (BdLV_1370), which also expresses $\triangle$ NGFR. The vector (schematised in Supplementary Fig. 6) was kindly provided by Luigi Naldini and his collaborators. Infected cells were analysed by FACS to isolate populations with similar $\triangle$ NGFR expression as previously ${ }^{41}$. Samples were analysed by counting 100,000 events per replica using a MACSQuant Flow cytometer (Miltenyi). Data analysis was performed by FlowJo software (TreeStar): fluorescent levels of $\triangle$ NGFR and dGFP for each cell were log transformed and divided in 100 bins (each of 1000 cells) based on $\triangle$ NGFR level. The mean $\triangle$ NGFR or dGFP expression was calculated in each bin and repression folds were calculated, at similar $\triangle$ NGFR mean expression, as the ratio: [dGFP(miR-30)/ [dGFP(control)].

miR-30c-5p sensor sequence:

TCTAGATAAGCTGAGAGTGTAGGATGTTTACACGATGCTGAGAGTGT AGGATGTTTACAACGCGTGTCGACGCTGAGAGTGTAGGATGTTTACATC ACGCTGAGAGTGTAGGATGTTTACACCCGGG

Control (scrambled) sensor sequence

FW TCTAGAGGAGCTCCACCGCGGTGGCATC

REV CCGGGATGCCACCGCGGTGGAGCTCCT

Data availability. The small RNA sequencing (sRNA-seq) and the RNAsequencing data set for this study have been submitted to the NCBI Gene Expression Omnibus (GEO; http://www.ncbi. nlm.nih.gov/geo/) under the accession number GSE104650. The 'serum stimulation data set', which contains miRNA expression analysis of WT cells, was previously submitted under accession number GSE72655. Data within the manuscript is available from the authors upon reasonable request.

Received: 4 December 2017 Accepted: 19 June 2018

Published online: 07 August 2018

\section{References}

1. Jonas, S. \& Izaurralde, E. Towards a molecular understanding of microRNAmediated gene silencing. Nat. Rev. Genet. 16, 421-433 (2015).

2. Bartel, D. P. MicroRNAs: target recognition and regulatory functions. Cell 136, 215-233 (2009).

3. Hafner, M. et al. Transcriptome-wide identification of RNA-binding protein and microRNA target sites by PAR-CLIP. Cell 141, 129-141 (2010).

4. Chi, S. W., Zang, J. B., Mele, A. \& Darnell, R. B. Argonaute HITS-CLIP decodes microRNA-mRNA interaction maps. Nature 460, 479-486 (2009).

5. Huntzinger, E. \& Izaurralde, E. Gene silencing by microRNAs: contributions of translational repression and mRNA decay. Nat. Rev. Genet. 12, 99-110 (2011).

6. Eulalio, A., Huntzinger, E. \& Izaurralde, E. Getting to the root of miRNAmediated gene silencing. Cell 132, 9-14 (2008).

7. Friedman, R. C., Farh, K. K., Burge, C. B. \& Bartel, D. P. Most mammalian mRNAs are conserved targets of microRNAs. Genome Res. 19, 92-105 (2009).

8. Hausser, J. \& Zavolan, M. Identification and consequences of miRNA-target interactions--beyond repression of gene expression. Nat. Rev. Genet. 15, 599-612 (2014).

9. Baek, D. et al. The impact of microRNAs on protein output. Nature $\mathbf{4 5 5}$, 64-71 (2008)

10. Selbach, M. et al. Widespread changes in protein synthesis induced by microRNAs. Nature 455, 58-63 (2008)

11. Thomson, D. W., Dinger, M. E. Endogenous microRNA sponges: evidence and controversy. Nat. Rev. Genet. 17, 273-283 (2016).

12. Ruegger, S. \& Grosshans, H. MicroRNA turnover: when, how, and why. Trends Biochem. Sci. 37, 436-446 (2012).

13. Salmena, L., Poliseno, L., Tay, Y., Kats, L. \& Pandolfi, P. P. A ceRNA hypothesis: the Rosetta Stone of a hidden RNA language? Cell 146, 353-358 (2011).

14. Bosson, A. D., Zamudio, J. R. \& Sharp, P. A. Endogenous miRNA and target concentrations determine susceptibility to potential ceRNA competition. Mol. Cell 56, 347-359 (2014).
15. Denzler, R., Agarwal, V., Stefano, J., Bartel, D. P. \& Stoffel, M. Assessing the ceRNA hypothesis with quantitative measurements of miRNA and target abundance. Mol. Cell 54, 766-776 (2014).

16. Denzler, R. et al. Impact of MicroRNA levels, target-site complementarity, and cooperativity on competing endogenous RNA-regulated gene expression. Mol. Cell 64, 565-579 (2016)

17. Bitetti, A. et al. MicroRNA degradation by a conserved target RNA regulates animal behavior. Nat. Struct. Mol. Biol. 25, 244-251 (2018).

18. de la Mata, M. et al. Potent degradation of neuronal miRNAs induced by highly complementary targets. EMBO Rep. 16, 500-511 (2015).

19. Ameres, S. L. et al. Target RNA-directed trimming and tailing of small silencing RNAs. Science 328, 1534-1539 (2010).

20. De, N. et al. Highly complementary target RNAs promote release of guide RNAs from human Argonaute2. Mol. Cell 50, 344-355 (2013).

21. Park, J. H., Shin, S. Y. \& Shin, C. Non-canonical targets destabilize microRNAs in human Argonautes. Nucl. Acids Res. 45, 1569-1583 (2017).

22. Cazalla, D., Yario, T. \& Steitz, J. A. Down-regulation of a host microRNA by a Herpesvirus saimiri noncoding RNA. Science 328, 1563-1566 (2010).

23. Marcinowski, L. et al. Degradation of cellular mir-27 by a novel, highly abundant viral transcript is important for efficient virus replication in vivo. PLoS Pathog. 8, e1002510 (2012).

24. Baccarini, A. et al. Kinetic analysis reveals the fate of a microRNA following target regulation in mammalian cells. Curr. Biol. CB 21, 369-376 (2011).

25. Krol, J. et al. Characterizing light-regulated retinal MicroRNAs reveals rapid turnover as a common property of neuronal microRNAs. Cell 141, 618-631 (2010).

26. Rissland, O. S., Hong, S. -J. \& Bartel, D. P. MicroRNA destabilization enables dynamic regulation of the miR-16 family in response to cell-cycle changes. Mol. Cell 43, 993-1004 (2011).

27. Marzi, M. J. et al. Degradation dynamics of microRNAs revealed by a novel pulse-chase approach. Genome Res.26, 554-565 (2016).

28. Duffy, E. E. et al. Tracking distinct RNA populations using efficient and reversible covalent chemistry. Mol. Cell 59, 858-866 (2015).

29. Agarwal, V., Bell G. W., Nam J. W. \& Bartel D. P. Predicting effective microRNA target sites in mammalian mRNAs. Elife 4, e05005 (2015).

30. Iyer, V. R. et al. The transcriptional program in the response of human fibroblasts to serum. Science 283, 83-87 (1999).

31. Haas, G. et al. Identification of factors involved in target RNA-directed microRNA degradation. Nucl. Acids Res. 44, 2873-2887 (2016).

32. Muller, H., Marzi, M. J. \& Nicassio, F. IsomiRage: From functional classification to differential expression of miRNA isoforms. Front. Bioeng. Biotechnol. 2, 38 (2014).

33. Rabani, M. et al. Metabolic labeling of RNA uncovers principles of RNA production and degradation dynamics in mammalian cells. Nat. Biotechnol. 29, 436-442 (2011).

34. Ran, F. A. et al. Genome engineering using the CRISPR-Cas9 system. Nat. Protoc. 8, 2281-2308 (2013).

35. Vlachos, I. S. et al. DIANA-TarBase v7.0: indexing more than half a million experimentally supported miRNA:mRNA interactions. Nucl. Acids Res. 43, D153-D159 (2015)

36. Moore, M. J. et al. miRNA-target chimeras reveal miRNA 3'-end pairing as a major determinant of Argonaute target specificity. Nat. Commun. 6, 8864 (2015).

37. Helwak, A., Kudla, G., Dudnakova, T. \& Tollervey, D. Mapping the human miRNA interactome by CLASH reveals frequent noncanonical binding. Cell 153, 654-665 (2013).

38. Yang, S. J. et al. The miR-30 family: Versatile players in breast cancer. Tumour Biol. 39, 1010428317692204 (2017).

39. $\mathrm{Li}, \mathrm{J}$. et al. miR-30 regulates mitochondrial fission through targeting p53 and the dynamin-related protein-1 pathway. PLoS Genet. 6, e1000795 (2010).

40. Feillet, C. et al. Phase locking and multiple oscillating attractors for the coupled mammalian clock and cell cycle. Proc. Natl Acad. Sci. USA 111, 9828-9833 (2014)

41. Brown, B. D. et al. Endogenous microRNA can be broadly exploited to regulate transgene expression according to tissue, lineage and differentiation state. Nat. Biotechnol. 25, 1457-1467 (2007).

\section{Acknowledgements}

We thank the Genomic Unit (Luca Rotta, Salvatore Bianchi, Thelma Capra) for sequencing runs and Claudia Crovace for manuscript editing. This work was supported by grants from the Associazione Italiana per la Ricerca sul Cancro (AIRC) to F.N. (IG14085 and IG18774), Cariplo (2015-0590) to F.N and Cariplo (2016-0615) to M.J.M.

\section{Author contributions}

F.G. generated MRE-KO cells. F.G. and C.R performed characterisation of precursor and mature miRNAs, generated adenoviruses and performed reconstitution experiments. F. G., C.R., M.C. performed biological characterisation of MRE-KO cells. Samples for 
sequencing were prepared by M.J.M. and F.G., F.N., M.J.M. and I.S. performed bioinformatics and statistical analyses. M.J.M. and F.N. analysed data and wrote the manuscript.

\section{Additional information}

Supplementary Information accompanies this paper at https://doi.org/10.1038/s41467018-05182-9.

Competing interests: The authors declare no competing interests.

Reprints and permission information is available online at http://npg.nature.com/ reprintsandpermissions/

Publisher's note: Springer Nature remains neutral with regard to jurisdictional claims in published maps and institutional affiliations. (c) (i) Open Access This article is licensed under a Creative Commons Attribution 4.0 International License, which permits use, sharing, adaptation, distribution and reproduction in any medium or format, as long as you give appropriate credit to the original author(s) and the source, provide a link to the Creative Commons license, and indicate if changes were made. The images or other third party material in this article are included in the article's Creative Commons license, unless indicated otherwise in a credit line to the material. If material is not included in the article's Creative Commons license and your intended use is not permitted by statutory regulation or exceeds the permitted use, you will need to obtain permission directly from the copyright holder. To view a copy of this license, visit http://creativecommons.org/ licenses/by/4.0/.

(C) The Author(s) 2018 